\title{
Boundedness Below for Fermion Model Theories Part II. The Linear Lower Bound*
}

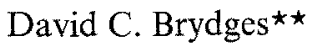 \\ University of Michigan, Department of Mathematics, Ann Arbor, Michigan 48104, USA
}

\begin{abstract}
The Generalised Yukawa Model $\left(G Y_{2}\right), \pm \bar{\psi} \psi \phi^{\mathrm{N}}+\phi^{2 \mathrm{M}}, \mathrm{M}>\mathrm{N}>1$, is bounded below, uniformly in a momentum cutoff, and linearly in the volume of a spatial cutoff.
\end{abstract}

\section{Introduction}

A lower bound, linear in the interaction volume and uniform in a momentum cutoff, is proven for the Hamiltonian associated with the Generalised Yukawa $\left(G Y_{2}\right)$ interaction $\left( \pm \bar{\psi} \psi \phi^{\mathrm{N}}+\phi^{2 \mathrm{M}}\right), \mathrm{M}>\mathrm{N}>1$, in two space-time dimensions. Thus, let $H$ denote the cutoff Hamiltonian as in (2.1), given below, then:

\section{Theorem.}

$$
H \geqq- \text { const ( } \operatorname{supp} g+1)
$$

where $g$ is the spatial cutoff (see 2.3) and the constant is uniform in the momentum cutoff.

The case $\mathrm{M}=\mathrm{N}=1$, the Yukawa model $\left(Y_{2}\right)$ was originally bounded below by Schrader [13]. The following proof can be extended to cover the Yukawa model with simple modifications, as in [5]. The proof is by expansion techniques analogous to those used by Glimm and Jaffe [8] in $P(\phi)_{2}$. These techniques have been developed for fermion fields by Federbush, and to a small extent, the author in $[1,4,5]$.

Another approach to this and other problems in Yukawa field Theories is contained in [14-16]. These authors obtain a full Euclidean Theory by integrating out the fermions. Their method is certainly neater than the one used in this paper and it is more effective for certain applications, e.g., $\phi$ bounds.

* This article includes the major part of a doctoral dissertation submitted in partial fulfillment of the requirements for the Doctor of Philosophy degree in the Horace H. Rackham School of Graduate Studies at the University of Michigan.

$\star \star$ Junior Fellow, Michigan Society of Fellows. 
While the $G Y_{2}$ model has no particular intrinsic interest, it provides a useful testing ground for ideas that have further applications. (See [2,3].) It is also expected that "cluster expansions" for field theories and statistical mechanical systems with fermions can be developed within the present time dependant Hamiltonian approach [3.1]. This paper is a continuation of [5], but can be real independantly.

The lower bound is obtained by estimating from below,

$$
-\lim _{T \rightarrow \infty} T^{-1} \log \left\langle e^{-T H}\right\rangle
$$

uniformly in a momentum cutoff and the expectation state. To make the calculation more transparent, (1.1) is first estimated for an expectation in the Fock vacuum. The simple changes needed to convert the calculation to obtain a bound uniform in the expectation are given in Appendix III.

The expression (1.1) is estimated by a type of perturbation expansion. Since the conventional perturbation series is divergent, the expansion is truncated by using a (logarithmic) divergent lower bound in a similar way to the use of the Wick lower bound in [8]. The substitute for a positivity preserving semigroup is the possibility of taking the operator norm over the fermion Fock space at a single point in $Q$ space. (The total Fock space is fibered over Nelson space.) This is called "defermiation".

The divergent lower bound is derived in Sections 6 and 7. The interaction is divided into two parts which are referred to as the "pair creation and annihilation" and "scattering" parts. The former is estimated by a modification of Glimm's dressing transformation [6] in which only fermions are dressed. (See [1].) The point of this is to obtain a lower bound holding pointwise with respect to $Q$ space. It also displays the essential idea behind the dressing transformation more clearly than in [6]. However, this dressing transformation is not as accurate as Glimm's and will not bound $Y_{2}$ or $G Y_{2}$ uniformly in a momentum cutoff. The lower bound provided by Section 6 involves nonlocal polynomials in the boson field raised to fractional powers. Section 7 extends Nelson's original proof of boundedness below for $P(\phi)_{2}$ to bound these terms. Here the presence of the $\phi^{2 M}$ with $M>N$ in the $G Y_{2}$ interaction is essential. Although the Yukawa model $Y_{2}$ is a special case of $M=N$ and can be obtained by modifications of the present proof, these methods do not suggest that the $\mathrm{M}=\mathrm{N}$ case is bounded below in general.

\subsection{Notation}

The total Hamiltonian is

$$
H=H_{0 B}+H_{0 F}+V
$$

where $H_{O B}, H_{O F}$ are the usual free field Hamiltonians. $V$ is the interaction

$$
V=\int g(x): \bar{\psi}_{\kappa} \psi_{\kappa}(x):: \phi^{N}(x): d x+\int h(x): \phi^{2 M}(x): d x-\Lambda .
$$

The subscript $\kappa$ on the $\psi s$ is to indicate that they have been cut off, i.e., $\psi_{\kappa}(x)=$ $(2 \pi)^{-1 / 2} u * \psi(x)$ where $u \in C^{\infty}(\mathbb{R})$ and the fourier transform $\hat{u}$ satisfies $|\hat{u}| \leqq 1$, $\hat{u}(p)=0$ for $|p| \geqq \kappa$. All estimates in this paper will be uniform is this cutoff. The 
subscript $\kappa$ will be suppressed. $g$ and $h$ are differentiable functions with compact support. $h$ is positive. In addition $g$ and $h$ are selected from the class of functions satisfying

$|g|,\left|g^{\prime}\right|, h,|g / h| \leqq c$

for some constant $C$ fixed in advance.

The counterterms, $A$, are

$$
A=\frac{1}{2} \sum_{j=0}^{N-1} \delta m_{j}^{2} \int g^{2}(x): \phi^{2(N-j)}(x): d x+E
$$

where

$$
\begin{aligned}
\delta m_{j}^{2}= & -2 \int_{\substack{p_{1}+p_{2}+k_{1}+\\
\ldots+k_{j}=0}}\left|\mathscr{K}_{p}\left(p_{1}, p_{2}\right)\right|^{2}\left(\omega_{1}+\omega_{2}+\mu_{1}+\ldots \mu_{j}\right)^{-1} \\
& \cdot\left(4 \pi \mu_{1}\right)^{-1} \ldots\left(4 \pi \mu_{j}\right)^{-1} .
\end{aligned}
$$

The integration is over the hyperplane specified beneath the integral sign.

$$
\begin{aligned}
E= & -\sum_{\Delta} \int\left|\left(g \chi_{\Delta}\right)\left(p_{1}+p_{2}+k_{1}+\ldots+k_{N}\right) \mathscr{K}_{p}\left(p_{1}, p_{2}\right)\right|^{2} \\
& \cdot\left(\omega_{1}+\omega_{2}+\mu_{1}+\ldots+\mu_{N}\right)^{-1}\left(4 \pi \mu_{1}\right)^{-1} \ldots\left(4 \pi \mu_{N}\right)^{-1} d p_{1} d p_{2} \\
& \cdot d k_{1} \ldots d k_{N}
\end{aligned}
$$

where $\omega_{1}=\omega\left(p_{1}\right), \omega_{2}=\omega\left(p_{2}\right)$ with $\omega(p)=\left(M^{2}+P^{2}\right)^{1 / 2}, \mu_{i}=\mu\left(k_{i}\right)$ with $\mu(k)=$ $\left(m^{2}+k^{2}\right)^{1 / 2} . \Delta$ and $\chi_{A}$ are defined in the next paragraph and

$$
\mathscr{K}_{p}=\left(\left(\omega_{1} \omega_{2}-p_{1} p_{2}-M^{2}\right) /\left(4 \pi \omega_{1} \omega_{2}\right)\right)^{1 / 2} \operatorname{sgn}\left(p_{1}-p_{2}\right) u\left(p_{1}\right) u\left(p_{2}\right)
$$

$M \equiv$ fermion mass. For future use, define also

$$
\begin{aligned}
& \mathscr{K}_{s}=\left(\left(\omega_{1} \omega_{2}+p_{1} p_{2}+M^{2}\right) /\left(4 \pi \omega_{1} \omega_{2}\right)\right)^{1 / 2} u\left(p_{1}\right) u\left(p_{2}\right) \\
& S\left(p_{1}, t\right)=(2 \pi)^{-3 / 2} \int u^{2}\left(p_{1}\right)(p+M)^{-1} e^{i p_{0} t} d p_{0}
\end{aligned}
$$

with $\gamma_{0}, \gamma_{1}$ as defined in [9], $\not \equiv p_{0} \gamma_{0}+i p_{1} \gamma_{1}$ so that $S$ represents the propagator (cutoff by $u^{2}$ ) continued to imaginary time.

\subsection{Spatial Cutoffs}

Throughout the paper $\Delta$ will denote a unit square belonging to a lattice of squares $\mathbb{R}^{2}$ with one square in the lattice centered on the origin. $\chi_{A}$ will denote the characteristic function of $\Delta . n(\Delta)$ will denote the number of vertices in $\Delta$, excluding $C$ vertices in $R$ terms. See under the heading 'expansion' for the meaning of these terms.

\subsection{Momentum Cutoffs}

Set $\kappa_{n}=n^{\alpha}$ where $\alpha$ will be chosen large to make the expansion converge. Let $\xi \in C_{0}^{\infty}(\mathbb{R})$, with $|\xi| \leqq 1$, be such that $\xi(p)=1$ for $|p| \leqq \frac{1}{2}, \xi(p)=0$ for $|p| \geqq 1$. The term "upper cutoff at $\kappa_{n}$ " means that the momentum space kernel : $\hat{\bar{\psi}}\left(p_{1}\right) \hat{\psi}\left(p_{2}\right)$ : is 
multiplied by $\varrho\left(p_{1}, p_{2}\right) \equiv \xi\left(p_{1} / \kappa_{n}\right) \xi\left(p_{2} / \kappa_{n}\right)$. Thus in configuration space

$$
: \bar{\psi} \psi(x): \mapsto(2 \pi)^{-1 / 2}\left[\int_{p_{1}+p_{2}=:} \varrho\left(p_{1}, p_{2}\right): \hat{\bar{\psi}}\left(p_{1}\right) \hat{\psi}\left(p_{2}\right):\right]^{\vee}(x)
$$

$\because$, represent the Fourier transform and its inverse. In (2.9) the "." denotes the variable being fourier transformed by " ". No cutoffs are put on boson momenta until Section 7. The term "lower cutoff at $\kappa_{n}$ " means that the function $\varrho\left(p_{1}, p_{2}\right)=$ $\left(1-\xi\left(p_{1} / \kappa_{n}\right) \xi\left(p_{2} / \kappa_{n}\right)\right.$ is substituted for $\varrho$ in $(2.9)$.

In the sequel, there will arise time dependent momentum cutoffs $\varrho \equiv \varrho(\tau) \equiv$ $\varrho\left(p_{0} p_{2}\right) \equiv \varrho\left(\tau_{1} p_{1}, p_{2}\right)$. These are always step functions of time and each step is either an upper or a lower momentum cutoff as above. The letter $\varrho$ will be used to denote all these types of cutoff.

The expansion will lower the momentum cutoff in the exponent of (1.1) in individual squares $\Delta$; therefore given a cutoff $\varrho$, which may depend on $\Delta$, define,

$$
\begin{gathered}
V(A, \tau)=\int: \phi_{A}:(x, \tau)(2 \pi)^{-1 / 2}\left[\int_{p_{1}+p_{2}=\cdot} \varrho: \hat{\bar{\psi}}\left(p_{1}\right) \hat{\psi}\left(p_{2}\right): d x\right]^{\nu} \\
+\int h \chi_{A}(x, \tau): \phi^{2 \mathrm{M}}:(x, \tau) d x-A(\Delta)
\end{gathered}
$$

where

$$
: \phi_{\Delta}^{N}:(x, \tau)=g(x) \chi_{\Delta}(x, \tau): \phi^{N}:(x, \tau)
$$

with $\phi(x, \tau)$ a Nelson boson field. Also, let

$$
\begin{aligned}
A(\Delta, \tau)= & \sum_{j=0}^{N-1} \frac{1}{2} \delta m_{j, \varrho}^{2} \int: \phi^{2 N-2 j}:(x, \tau) g^{2}(x) \chi_{A}(x, \tau) d x d \tau \\
& +E_{\varrho}(A, \tau)
\end{aligned}
$$

where

$$
\begin{aligned}
\delta m_{j, \ell}^{2}= & -2 \int_{p_{1}+p_{2}+k_{1}+}\left|\mathscr{K}_{p}\left(p_{1}, p_{2}\right)\right|^{2} \varrho^{2}\left(p_{1}, p_{2}\right) \\
& \cdot\left(\omega_{1}+\omega_{2}+\mu_{1}+\ldots+\mu_{j}\right)^{-1}\left(4 \pi \mu_{1}\right)^{-1} \ldots\left(4 \pi \mu_{j}\right)^{-1} \\
E_{\varrho}(A, \tau)= & -\int\left|\left(g \chi_{\Delta}\right)^{\prime}\left(p_{1}+p_{2}+\ldots k_{N}\right)\right|^{2} \varrho^{2}\left(p_{1}, p_{2}\right) \mathscr{K}_{p}^{2}\left(p_{1}, p_{2}\right) \\
& \cdot\left(\omega_{1}+\omega_{2}+\mu_{1}+\ldots+\mu_{N}\right)^{-1}\left(4 \pi \mu_{1}\right)^{-1} \ldots\left(4 \pi \mu_{N}\right)^{-1} d p_{1} \ldots d k_{N} .
\end{aligned}
$$

\section{Miscellaneous}

The symbol $O(1)$ is used to indicate constants which are uniform in the variables of interest. The energy counterterm given in (2.6) differs from the usual one by a nondivergent quantity linear in the volume. Fourier transforms and partial fourier transforms are indiscriminately notated by $\hat{,}, ;$; the context is supposed to supply the missing information. " denotes the inverse fourier transform to .

\section{The Expansion}

Following the procedure in [5], $\left\langle e^{-T H}\right\rangle$ is rewritten in terms of Nelson boson fields, via the Trotter Product formula.

$$
\left\langle e^{-T H}\right\rangle=\lim _{N \rightarrow \infty} \pi\left\langle\prod_{J=1}^{N} e^{-\left[H_{0 F}(j T / N)+V(j T / N)\right]}\right\rangle .
$$


The expectation for the boson fields is with respect to the Nelson Fock vacuum on the right hand side. The $\mathbb{T}$ stands for time ordering and for this purpose the fermion fields are to be given a dummy time dependance, hence the time argument in $H_{0 F}$ and in $V$ in (3.1) and (3.2). In order to display the algebraic structure of the expansion a formal rearrangement of (3.1) is used:

$$
\left\langle e^{-T H}\right\rangle=\mathbb{T}\left\langle e^{-\int_{0}^{T} H_{0 F}(\tau) d \tau} e^{-\int_{0}^{T} V(\tau) d \tau}\right\rangle .
$$

Expressions like (3.2) are to be regarded merely as a convenient shorthand for limits of time ordered products as in (3.1).

The identities which generate the expansion are now given.

$$
\begin{aligned}
& \mathbb{T}\left\langle e^{-\int_{0}^{T} H_{O F}(\tau) d \tau} e^{-\int_{0}^{T} V^{\prime}(\tau) d \tau}\right\rangle \\
= & \mathbb{T}\left\langle e^{-\int_{0}^{T} H_{0 F}(\tau) d \tau} e^{-\int_{0}^{T} V^{\prime \prime}(\tau) d \tau}\right. \\
& -\int_{0}^{T} d s \mathbb{T} e^{-\int_{0}^{T} H_{0 F}(\tau) d \tau} e^{-\int_{0}^{T} V^{\prime}(\tau) d \tau}\left(V^{\prime}-V^{\prime \prime}\right)(s) \\
& \left.\cdot e^{-\int_{0}^{s} V^{\prime \prime}(\tau) d \tau}\right\rangle .
\end{aligned}
$$

$V^{\prime}$ and $V^{\prime \prime}$ are being used to denote sums over $\Delta$ of terms of the form (2.10). Again, in a formal sense (3.3) can be summarised by:

$$
e^{-\int_{0}^{T} V^{\prime}}=e^{-\int_{0}^{T} V^{\prime \prime}}-\int_{0}^{T} d s\left(V^{*}-V^{\prime \prime}\right)(s) e^{-\int_{s}^{T} V^{\prime}-\int_{0}^{s} V^{\prime \prime}} .
$$

In the expansion, (3.4) is used to lower the momentum cutoffs in individual space-time squares. Thus write

$$
g(x)=\sum_{\Delta} g(x) \chi_{\Delta}(x, s) .
$$

Note that the right hand side is independent of the time variable s. Correspondingly

$$
V^{\prime}=\sum_{\Delta} V^{\prime}(\Delta)
$$

where the cutoff in each $\Delta$ will in general vary with $\Delta$. (3.4) may be applied to alter the cutoff $\varrho^{\prime}$ in a single square $\Delta$ to $\varrho^{\prime \prime}$ by

$$
\begin{aligned}
e^{-\int V^{\prime}(\Delta)}= & e^{-f V^{\prime \prime}(\Delta)}-\int d s\left[V^{\prime}(\Delta, s)-V^{\prime \prime}(\Delta, s)\right] \\
& \cdot e^{-\int V^{\infty}(\Delta)-\int V^{\prime \prime}(\Delta)} .
\end{aligned}
$$

(3.7) is called the $P$ formula (for perturbation). The $\left(V^{\prime}-V^{\prime \prime}\right)(\Delta)$ will be referred to as a $P$ vertex. Note that the ranges of the time integrals in $(3.7)$ are restricted by the time localisation of the $V(\Delta) s$. 
The other identity used to obtain the expansion is called the contraction formula or $C$ formula. In the interest of brevity it is also stated formally. Let $\sigma_{1} \geqq \sigma_{2} . b(p)$ is a fermion annihilation operator.

$$
\begin{aligned}
b\left(p, \sigma_{1}\right) e^{-\delta V^{\prime} d \tau}= & e^{-\omega(p)\left(\sigma_{1}-\sigma_{2}\right)} b\left(p_{1}, \sigma_{2}\right) e^{-j V^{\prime} d \tau} \\
& -\int_{\sigma_{1}}^{\sigma_{2}} d \sigma\left[e^{-\omega(p)\left(\sigma_{1}-\sigma\right)} b(p, \sigma), V^{\prime}(\sigma)\right] e^{-\delta V^{\prime} d \tau} .
\end{aligned}
$$

Similar identities hold for $b^{\prime}$ and $b^{*}, b^{*}$. A monomial in fermion annihilation and creation operators $b^{*}$ at different times may also be included. (As will often be done in the sequel, any $\phi$ dependence will be absorbed into the kernel.) Suppose $R=R\left(b^{\#}\left(s_{1}\right), b^{\#}\left(s_{2}\right), \ldots, b^{\#}\left(s_{n}\right)\right)$ is such an object, then (3.8) is modified to,

$$
\begin{aligned}
& b\left(p, \sigma_{1}\right) R e^{-\rho V^{\prime}}=e^{-\omega\left(\sigma_{1}-\sigma_{2}\right)} b\left(p, \sigma_{2}\right) R e^{-\delta V^{\prime}} \\
& \quad-R \int_{\sigma_{1}}^{\sigma_{2}} d \sigma\left[e^{-\omega\left(\sigma_{1}-\sigma\right)} b(p, \sigma), V^{\prime}(\sigma)\right] e^{-\rho V^{\prime}} \\
& \quad+\sum_{i=1}^{n}(-1)^{i} R\left(b^{\#}\left(s_{1}\right), \ldots\left\{e^{-\omega\left(\sigma_{1}-s_{i}\right)} b\left(p, s_{i}\right), b^{\#}\left(s_{i}\right)\right\}, \ldots, b^{\#}\left(s_{n}\right)\right) e^{-\delta V^{\prime}} .
\end{aligned}
$$

The second term in (3.9) will be referred to as a contraction to the exponent and the commutator therein as a $C$ vertex. In order to obtain the rigorous identities which (3.7) and (3.9) abbreviate, multiply by $e^{-j H_{0 F}(\tau) d \tau}$, time order, and take the Fock expectation. For time ordering understand a limit of stepwise time dependant quantities as in (3.1). The $b(p)$ in (3.8) and (3.9) is to be smeared with an $L^{2}$ function. The justification of these limits is omitted.

The expansion is now described. For a given $\Delta, n$, and times $s_{1} \leqq s_{2} \leqq \ldots \leqq s_{n} \in \Delta$ ( $s \in \Delta$ means $(x, s) \in \Delta$ for some $x$ ), let $\varrho \equiv \varrho(\tau)$ specify an upper cutoff at $\kappa_{i}$ for $s_{i-1}<\tau \leqq s_{i}$. (Set $s_{0}, s_{n+1}$ equal to the smallest and largest $s$ with $s \in \Delta$.) By (2.10) define the corresponding $V(\Delta) \equiv V\left(\Delta,\left(s_{i}\right), \tau\right) ; \quad\left(s_{i}\right)$ abbreviates $s_{0}, s_{1}, \ldots, s_{n+1}$. Let $\tilde{V}\left(\Delta,\left(s_{i}\right), \tau\right)$ be the same as $V\left(A,\left(s_{i}\right), \tau\right)$ except its cutoff $\varrho$ specifies an infinite upper cutoff for $\tau>s_{n}$.

Begin the expansion by selecting a square $\Delta$ and applying the $P$ formula (3.7) to (3.2) with $V^{\prime}(\Delta)=V(\Delta)$ with infinite upper cutoff and $V^{\prime \prime}(\Delta)=V(\Delta)$ with upper cutoff at $\kappa_{1}$. (See 2.10.) The $C$ formula (3.9) is now used to move the annihilation and creation operators of the $P$ vertex over to the vacuum where they annihilate. In the process, new $C$ vertices, contractions to the exponent, are formed. These are not further contracted. Both legs of the $P$ vertex are said to be contracted. Next, certain terms arising during contraction are cancelled. This is described under "renormalisation" below. All the terms which came from manipulations on the terms containing the $P$ vertex have an exponential dependance $e^{-f \tilde{V}\left(\Delta, s_{1}, \tau\right)}$ in the square $\Delta$. Equation (3.7) is applied to these terms with $V^{\prime}(\Delta)=\tilde{V}\left(\Delta, s_{1}, \tau\right)$ and $V^{\prime \prime}(\Delta)=V\left(\Delta, s_{1}, \tau\right)$. Again the resulting $P$ vertex is contracted and renormalised. Then (3.7) is applied etc. The $n$th application of (3.7) is with $V^{\prime}(\Delta)=\tilde{V}\left(\Delta, s_{1}, \ldots\right.$, $\left.s_{n-1}, \tau\right)$ and $V^{\prime \prime}(\Delta)=V\left(\Delta, s_{1}, \ldots, s_{n-1}, \tau\right)$. The expansion will terminate in $\Delta$ when $n$ is so large that $\kappa_{n}>\kappa$. Then a new square $A^{\prime}$ is chosen and expanded, and so on. 


\section{Renormalisation}

It is possible for a $P$ vertex to contract twice to the same $C$ vertex. Terms in which this occurs need special handling because they contribute divergences as $\kappa \rightarrow \infty$. They are to be cancelled against terms containing appropriate counterterms.

Write these terms as sums over the cases in which the $P$ and $C$ vertices are localised in squares $\Delta_{P}$ and $\Delta_{C}$. Terms for which $\Delta_{P} \neq \Delta_{C}$ do not give divergent contributions. This will be proven in appendix 1 . Therefore suppose $\Delta_{P}=\Delta_{C}=\Delta$. Let the times of the $P$ and $C$ vertices be $s_{P}$ and $s_{C}$. Let the $P$ vertex be the $\mathrm{n}^{\text {th }}$ $P$ vertex introduced into $\Delta . s_{n-1}$ denotes the time of the previous $P$ vertex introduced in $A$. Then the factor corresponding to the double contraction can be written as:

$$
\int d s_{C} d k\left(: \phi_{\Delta}^{N}: \hat{)}\left(k, s_{P}\right) r\left(k, s_{P}, s_{C}\right)\left(: \phi_{\Delta}^{N}:\right)^{\llcorner}\left(k, s_{C}\right)\right.
$$

where, if $\varrho_{P}$ and $\varrho_{C}$ are the cutoffs on the $P$ and $C$ vertices,

$$
\begin{aligned}
r\left(k_{g}, s_{P}, s_{C}\right)= & \int_{p_{1}+p_{2}=k} \mid \mathscr{K}_{p}\left(p_{1},\left.p_{2}\right|^{2} \varrho_{P}\left(p_{1}, p_{2}\right) \varrho_{C}\left(p_{1}, p_{2}, s_{C}\right)\right. \\
& \cdot e^{-\left(\omega_{1}+\omega_{2}\right)\left|s_{P}-s_{C}\right|} .
\end{aligned}
$$

Notice that since the cutoff in the exponent changes at the times of each $P$ vertex in $\Delta$, the cutoff, $\varrho_{C}$, on the $C$ vertex depends on $s_{C}$. Since the exponent does not depend on $s_{C}$, it is permissible to take it outside the integral over $s_{C}$ as is implicit in $(3.10)$.

Renormalisation consists of cancelling the term containing (3.10) against the term containing the right counterterm. This term is the same except that $(3.10)$ is replaced by [see (2.11)]

$$
\Lambda_{P}=\Lambda(\Delta, \tau)
$$

with cutoff $\varrho$ given by $\varrho^{2}=1-\varrho_{P}$.

It is convenient for the rest of the calculation to also group together the terms for which $\Delta_{P}$ and $\Delta_{C}$ are not the same but have a common side or corner. Thus let $\mathcal{N}\left(\Delta_{P}\right)$ be the set of all (9) $\Delta_{C} s$ with this property. Then on grouping together all the appropriate terms, one obtains a term with, associated with the $P$ vertex, a factor:

$$
\begin{aligned}
R= & \sum_{\Delta_{C} \in \mathscr{N}\left(\Delta_{P}\right)} \int d s_{C} d k\left(: \phi_{\Delta_{P}}^{N}:\right)^{\wedge}\left(k, s_{P}\right) r\left(k, s_{P}, s_{C}, \Delta_{C}\right) \\
& \cdot\left(: \phi_{\Delta_{C}}^{N}:\right)^{\sim}\left(k, s_{C}\right)-\Lambda_{P} .
\end{aligned}
$$

The dependence on $\Delta_{C}$ in $r$ is there because $\varrho_{C}$ depends on $\Delta_{C}$ in (3.11). Equation (3.13) will be referred to as an " $R$ term". The corresponding double contractions between vertices in the same or neighboring squares will be called " $R$ subgraphs".

Smoothing the uncontracted fermion fields:

One final operation is performed when the expansion, as described so far, is complete in every square; each uncontracted fermion field is contracted once more. This has the beneficial effect of incorporating the upper momentum cutoff in the exponent into every terminal vertex, i.e., a vertex with an uncontracted field. 
The expansion is written as

$$
\left\langle e^{-T H}\right\rangle=\sum_{\mathscr{g}} \int d s_{\mathscr{g}} \pi\left\langle K_{\mathscr{g}}\left(s_{\mathscr{g}}\right) e^{-\int d \tau H_{0 F}(\tau)} \prod_{\Delta} e^{-\int d \tau V\left(\Delta, s_{g}, \tau\right)}\right\rangle .
$$

$V\left(\Delta, s_{g}, \tau\right)=V\left(\Delta,\left(s_{i}\right), \tau\right)$ where $\left(s_{i}\right)$ are the times of the $P$ vertices for a given term indexed by $g . s_{g}$ denotes the set of times of all vertices occurring in the term except those in $R$ subgraphs which have already been integrated out. (See (3.13).) $g$ comprises (a) a function $\Delta \mapsto n^{\prime}(\Delta)$ specifying the number of vertices in $\Delta$. (b) for each $\Delta$, a function $\{1,2,3, \ldots, n(\Delta)\} \mapsto\{P, C\}$ which labels the vertices in $\Delta$ and specifies whether they be $P$ or $C$ vertices. (c) a graph on the vertices given by (a) and (b), which specifies the contractions arising from use of (3.9). Uncontracted fermion fields are represented by open lines, i.e., lines which leave a vertex but do not end on another vertex. Finally $g$ 's which are the same except for specifying different $R$ subgraphs for a given $P$ vertex are identified. Define $n(A)$ as the number of vertices in $\Delta$, excluding $C$ vertices in $R$ subgraphs, so that the equivalence classes of $g$ 's will have $n(\Delta)$ well defined for each $\Delta$.

Given $g$ and $s_{g}, K_{g}$ is obtained by the following prescriptions. Assign to each $P$ vertex in an $R$ subgraph, the factor $R$ given by (3.13). To each vertex $v$ at $s_{v}$ in $\Delta$ which is not part of an $R$ term, assign the factor $Q_{v}\left(p_{1}, p_{2}\right)\left(: \phi_{\Delta}^{N}:\right)^{\wedge}\left(p_{1}+p_{2}\right)$. If $v$ is the $n^{\text {th }} P$ vertex formed in $\Delta$ by the expansion, $\varrho_{v}$ specifies a lower cutoff at $\kappa_{n}$. $C$ vertices have an upper momentum cutoff whose form is not important except when $v$ is a $C$ vertex formed in smoothing the uncontracted legs. In that case $v$ has a time dependant cutoff less than $\kappa_{n(\Delta)}$. To each closed line assign a cutoff propagator $S$. See (2.8). To each open line assign either $\psi$ or $\bar{\psi}$ in a consistent way. Integrate over all momenta as indicated by the graph specified by $g$.

The time integrations for the $P$ vertices are over a time ordered region, (the order being that in which they were formed). $C$ vertices are not time ordered.

\section{Estimates}

To begin with, the fermion fields are removed from (3.14) by the lemma below, called "defermiation".

Define the kernel $\tilde{K}_{g}$ of $K_{g}$ by

$$
K_{g}=\int \sum \tilde{K}_{g}\left(p_{1}, \alpha_{1}, \ldots, p_{n}, \alpha_{n}\right) \psi_{\alpha_{1}}^{*}\left(p_{1}, s_{1}\right) \ldots \psi_{\alpha_{n}}^{*}\left(p_{n}, s_{n}\right)
$$

$\psi^{\#}$ denotes $\psi$ or $\bar{\psi}$. The sum is over the spinor indices $\alpha_{1}, \ldots, \alpha_{n}$. The integral is over the momenta $p_{i}$.

Lemma. Suppose there exists a function of Nelson fields $c(\phi)$ which may depend on $\tau, s_{y}$, such that as forms

$$
H_{0 F}(\tau)+\sum_{\Delta} V\left(A, s_{y}, \tau\right) \geqq-c(\phi)
$$

pointwise in $\tau$ and $s_{y}$, then

$$
\mathbb{T}\left\langle K_{g}\left(s_{g}\right) e^{-\int H_{O F}} \prod_{A} e^{-j V\left(A, s_{f}, \tau\right)}\right\rangle \leqq\left\langle\operatorname{Tr}\left|\tilde{K}_{g}\left(s_{g}\right)\right| e^{\int c(\phi) d \tau}\right\rangle .
$$

$\operatorname{Tr}\left|\tilde{K}_{g}\right|$ means the following. The arguments of $\tilde{K}_{g}$ come in pairs corresponding to the ends of subgraphs consisting of connected open lines in the total graph 
specified by $g . \tilde{K}_{g}$ is the kernel of an operator acting on the Hilbert space of $L^{2}$ functions of the first elements of these pairs. Identify $\tilde{K}_{g}$ with this operator, then $\left|\tilde{K}_{g}\right|$ means $\sqrt{K_{g}^{*} \bar{K}_{y}}$ and trace has its usual meaning. Lemma (4.2) is a simple consequence of the inequality $\left\|\psi^{\#}(f)\right\| \leqq\|f\|_{2}$. Write $\tilde{K}_{g}$ as a matrix with respect to pairs of orthogonal product bases, and choose an optimal set. A proof of a similar estimate is given in more detail in [5].

In Sections 6 and 7 it is shown that there exists a $c(\phi)$ satisfying (4.3) and

$$
\left\langle e^{2 \int f(\phi) d \tau}\right\rangle^{1 / 2} \leqq \prod_{\Delta} O(1)^{n(\Delta)}
$$

Lemma (4.4), (4.5) and the Cauchy inequality imply

$$
\left\langle e^{-T H}\right\rangle \leqq \sum_{\mathscr{g}} \int d s_{\mathscr{g}}\left\langle\left(\operatorname{Tr}\left|\tilde{K}_{g}\right|\right)^{2}\right\rangle^{1 / 2} \prod_{A} O(1)^{n(d)}
$$

The next task will be to estimate $\left.\left(\operatorname{Tr} \mid K_{g}\right)\right)^{2}$ by polynomials in Nelson fields. Basically this is done by applying the inequality, $n \geqq 2$,

$$
\operatorname{Tr}\left|Q_{1} B_{1} Q_{2} B_{2} \ldots Q_{n} B_{n}\right| \leqq\left\|Q_{1}\right\|_{\text {H.S. }}\left\|B_{1}\right\| \ldots\left\|Q_{n}\right\|_{\text {H.S. }}\left\|B_{n}\right\|
$$

with suitable choices of the operators $Q_{i}, B_{i}$. H.S. stands for the Hilbert Schmidt norm. However it is necessary to obtain estimates that take into account the small contributions that are made by $g$ 's which specify contractions between vertices in widely separated squares or vertices with large lower cutoffs. The estimates discussed below all hold pointwise with respect to $s_{g}$ which is to be regarded merely as a parameter.

For a given $s_{y}$, the cutoff on a vertex has the form

$$
\varrho\left(p_{1}, p_{2}\right)=\xi\left(p_{1} / \mu\right) \xi\left(p_{2} / \mu\right)\left(1-\xi\left(p_{1} / \lambda\right) \xi\left(p_{2} / \lambda\right)\right.
$$

where $\mu$ specifies the upper cutoff and $\lambda$ the lower. This may be rewritten in the form

$$
\xi\left(p_{1} / \mu\right)\left(1-\xi\left(p_{1} / \lambda\right)\right) \xi\left(p_{2} / \mu\right) \xi\left(p_{2} / \lambda\right)+\xi\left(p_{1} / \mu\right) \xi\left(p_{2} / \mu\right)\left(1-\xi\left(p_{2} / \lambda\right)\right) .
$$

Rewrite the kernel $\tilde{K}_{g}$ as a sum corresponding to the above splitting being made at each vertex. The momentum cutoff at a given vertex of a term in this sum has the form of a product of a function of $p_{1}$ only, with a function of $p_{2}$ only. These functions are to be absorbed into the propagators which is indicated by replacing $S$ by $S^{\prime}$ below. (See 2.8.) Let $l$ be a line joining two vertices. Let $d_{l}$ be the distance between the closest points of the squares within which each vertex is localised. Let $\chi \in C^{\infty}(\mathbb{R})$ be a function such that $\chi(t)=0$ if $t \leqq 1 / 2, \chi(t)=1$ if $t \geqq 1$, and define $X_{l} \in C^{\infty}\left(\mathbb{R}^{2}\right)$ by putting $\chi_{l}(x)=\chi\left(|x| / d_{l}\right) ; x \in \mathbb{R}^{2}$ denotes a point in space time. If $d_{l}=0$ set $\chi_{l}=1$. If $S^{\prime}=S^{\prime}(p)$ (keeping $s_{z}$ as a parameter) is the propagator corresponding to the line $l$, the replacement

$$
S^{\prime} \rightarrow \tilde{S} \equiv\left(\hat{S}^{\prime} \chi_{l}\right)^{-}
$$

changes nothing because the localisation of the vertices connected by $l$ is such that $\chi_{1}=1 . \tilde{S}$ satisfies two estimates (4.8) and (4.9).

$$
\left\|\omega^{\varepsilon-} \tilde{S} \omega^{\varepsilon+}\right\| \leqq O(1)(1+\lambda)^{-\eta} t_{l}^{-\eta-\varepsilon_{--}-\varepsilon_{+}} ; \quad \eta, \varepsilon_{-}, \varepsilon_{+}>0
$$


$\lambda$ specifies the lower cutoff which has been absorbed into the propagator. $t_{l}$ denotes the time difference between the vertices contracted by $l$. The norm sign means operator norm on $L^{2}(\mathbb{R}) \otimes$ spinor space, identifying $\omega$ and $\tilde{S}$ with the obvious operators on $p$-space. (4.8) is easy to prove. The other estimate is

$$
\|\omega \tilde{S} \omega\| \leqq O\left((1+\lambda)^{-\eta} d_{l}^{-m}\right) ; \quad d_{l}>0
$$

for all $m \geqq 0$ and some $\eta>0$. To prove (4.9):

$$
\left|\omega^{2} \tilde{S}_{\alpha \beta}\right| \leqq\left\|\left(M^{2}-\partial^{2} / \partial x^{2}\right) S_{\alpha \beta}^{\prime} \chi_{l}\right\|_{1} .
$$

The subscript is to indicate the $L^{1}$ norm. (4.9) is immediate, because of the rapid decay of $S^{\prime}$ and its derivatives away from the origin. The $(1+\lambda)^{-\eta}$ can be obtained by scaling.

Let $Q_{v}$ have the kernel

$$
\left(1+\lambda_{v}\right)^{-\eta} \omega^{-\varepsilon_{1}}\left(p_{1}\right)\left(: \phi_{\Delta}^{N}:\right)^{n}\left(p_{1}+p_{2}\right) \omega^{-\varepsilon_{2}}\left(p_{2}\right)
$$

where $\Delta$ is the square where vertex $v$ is localised. $\lambda_{v}$ is the lower cutoff on $v$. The choice of $\varepsilon_{1}, \varepsilon_{2}$ and $\eta$ will be made later. Choose the $B_{i}$ 's to have the kernel $\omega^{\varepsilon-} \tilde{S} \omega^{\varepsilon+}$ where $\varepsilon_{ \pm}$will depend on the line associated with $\tilde{S}$. All the subgraphs specified by $g$ are either closed loops or open connected lines. A closed loop corresponds to an expression with the form of a trace of a product of $Q$ 's and $B$ 's which may be majorised by (4.7). An open connected line also corresponds to such a product and its trace norm which appears in (4.6) can also be estimated by (4.7). Only the $R$ terms (see 3.13) are excluded from this procedure. They are majorised simply by their absolute value. Whenever a line has $d_{l}>0(4.9)$ is used on the corresponding $B$ (thus choose $\varepsilon_{ \pm}=1$ for such a $B$ ). If $d_{l}=0,(4.8)$ is used. Hence for $\eta>0$ sufficiently small,

$$
\left(\operatorname{Tr}\left|\tilde{K}_{g}\right|\right)^{2} \leqq \prod_{v, \mu} O(1)\left\|Q_{v}\right\|_{\text {H.S. }}\left|R_{\mu}\right| \prod_{l} t_{l}^{-\sigma_{l}}\left(1+d_{l}\right)^{-m}
$$

where $\mu$ runs over all $P$ vertices in $R$ terms and $v$ runs over the other vertices. $l$ runs over all lines in the graph except those in $R$ terms. $\sigma_{l}$ depends on the $\varepsilon$ 's and $\eta$, which are yet to be chosen. Given $l$ with $d_{1}>0, \sigma_{l}=0$. If $d_{l}=0$ then $\sigma_{l}=\varepsilon_{-}+\varepsilon_{+}+\eta$ where,-+ label the ends of $l$. Equation (4.12) is valid for all $m \geqq 0$.

The next lemma will be used to estimate $\left\langle\prod_{\nu, \mu}\left\|Q_{v}\right\|_{\text {H.S. }}^{2} R_{\mu}^{2}\right\rangle$.

Lemma. Let $L$ be a lattice of squares on $\mathbb{R}^{2}$. Suppose $f_{v}, v=1,2, \ldots$ are functions on $Q$ space, each one belonging to an algebra generated by Nelson fields supported in a square in $L$; then for some $\beta$ independent of $n$,

$$
\left|\left\langle\prod_{v} f_{v}\right\rangle\right| \leqq \prod_{A \in L}\left\|\prod_{v \in A} f_{v}\right\|_{\beta} .
$$

By abuse of notation $v \in \Lambda$ is to indicate that the $f_{v}$ belongs to the local algebra generated by fields supported in $A$. \|\|$_{\beta}$ means the norm on $L_{\beta}(Q)$.

Proof. This is the "Sandwich Estimate" in [12]. Briefly, the proof goes as follows: Suppose that $h$ is in a local algebra generated by fields supported in a strip with parallel sides and let $E, E^{\prime}$ project onto functions in the two algebras 
generated by fields supported on the edges. Then by hypercontractivity, [12],

$$
\left\|E h E^{\prime}\right\| \leqq\|h\|_{\beta^{\prime}}
$$

for some $\beta^{\prime}$ depending only on the width of the strip. || || means the operator norm on $L^{2}(Q)$. To obtain (4.13), order the $f_{v}$ by the horizontal abscissa of their supports. Insert projections onto vertical lines using the Markov property, then use (4.14). The left hand side of (4.13) has now been dominated by $L_{\beta^{\prime}}$ norms of functions supported in vertical strips. Apply the same argument, but using projections onto horizontal lines, to each vertical strip to obtain (4.13).

The lemma is not immediately applicable to $\left\langle\prod_{\nu, \mu}\left\|Q_{v}\right\|_{\text {H.S. }}^{2} R_{\mu}^{2}\right\rangle^{1 / 2}$ because the $R_{\mu}$ are supported in overlapping $3 \times 3$ squares. To remove the overlap, form 9 lattices of $3 \times 3$ squares $L_{1}, \ldots, L_{q}$ by unit translations, horizontally or vertically, of a $3 \times 3$ lattice centred on the origin. Correspondingly partition the set of $v$ 's and $\mu$ 's into $G_{1}, \ldots, G_{q}$ so that $v$ belongs to $G_{m}$ if $v \in \Delta$ with $\Delta$ the central unit square of a $3 \times 3$ square in $L_{m}, \mu \in G_{m}$ if the fields of $R_{\mu}$ are supported in a $3 \times 3$ square belonging to $L_{m}$. This is tantamount to saying that the $P$ vertex of $R_{\mu}$ belongs to a $A$ as above. Then by the Holder inequality:

$$
\left\|\prod_{v, \mu}\right\| Q_{v}\left\|R_{\mu}\right\|_{2} \leqq \prod_{m=1}^{q}\left\|\prod_{v, \mu \in \boldsymbol{G}_{m}}^{l}\right\| Q_{v}\left\|_{\mathrm{H.S} .} R_{\mu}\right\|_{18} .
$$

Apply (4.13) to each $L_{18}$ norm using $3 \times 3$ squares $A$. Thus the left hand side of (4.15) is dominated by

$$
\prod_{m=1}^{q} \prod_{\substack{A \in L_{m} \\ \|}}\left\|\prod_{\substack{v, \mu \in A \\ v, \mu \in G_{m}}}\right\| Q_{v}\left\|_{\text {H.S. }} R_{\mu}\right\| \|
$$

for some $\gamma$ independant of the total number of $v$ 's and $\mu$ 's. By the Holder inequality (4.16) is less than

$$
\prod_{\Delta} \prod_{y, u \in \Delta}\|\| Q_{v}\left\|_{\text {H.S. }}\right\|_{\gamma n(\Delta)}\left\|R_{\mu}\right\|_{\gamma n(\Delta)}
$$

where $\Delta$ is a unit square as usual. This is because the number of $v$ 's and $\mu$ 's belonging to a given $A$ and $G_{m}$ is $n(A) .\left\|Q_{v}\right\|_{\text {H.S. }}^{2}$ and $R_{\mu}$ are polynomials in Nelson fields. It is a simple consequence of Nelson's Best Estimate (see [11]) applied to $e^{-t N}$ where $N$ is the number operator that

$$
\begin{aligned}
& \|\| Q_{v}\left\|_{\text {H.S. }}^{2}\right\|_{p} \leqq p^{N}\left\|Q_{v}\right\|^{2} ; \quad\left\|Q_{v}\right\|^{2} \equiv\|\| Q_{v}\|\|_{2} \\
& \left\|R_{\mu}\right\|_{p} \leqq p^{N}\left\|R_{\mu}\right\|_{2} .
\end{aligned}
$$

The $N$ in (4.18) is the $N$ in the definition of the $(G Y)_{2}$ interaction. (4.18) applied to (4.17) shows that

$$
\left\|\prod_{v, \mu}\right\| Q_{v}\left\|_{\text {H.S. }} R_{\mu}\right\|_{2} \leqq \prod_{\Delta}[O(1) n(\Delta)]^{N n(\Delta)} \prod_{v, \mu}\left\|Q_{v}\right\|\left\|R_{\mu}\right\|_{2} .
$$




\section{Convergence of the Expansion}

Equations (4.19), (4.12), and (4.6) imply,

$$
\begin{aligned}
\left\langle e^{-T H}\right\rangle \leqq & \sum_{g}\left[\prod_{A}(O(1) n(A))^{N n(\Delta)}\right] \\
& \cdot \int d s_{g} \prod_{v, \mu}\left\|Q_{v}\right\|\left\|R_{\mu}\right\|_{2} \prod_{l}\left(1+d_{l}\right)^{-m} t_{l}^{-\sigma_{i}} .
\end{aligned}
$$

It is now a purely numerical (as opposed to operator) problem to verify that (5.1) converges for suitable choices of $\alpha,\left(\kappa_{j}=j^{\alpha}\right), m, \eta$ and the $\varepsilon^{\prime}$ s in (4.11). The order in which these are to be chosen is: $\varepsilon$ 's and $\eta, \alpha, m$. Three estimates, (5.2), (5.3), and (5.4) will be used. (5.2) and (5.3) are not hard to prove, so proofs are not given. Estimate (5.4) is discussed in the appendix.

$$
\begin{aligned}
& \text { If } \varepsilon_{1}>0, \varepsilon_{2}>0 \text { and } \varepsilon_{1}+\varepsilon_{2}>1 / 2 \\
& \left\|Q_{v}\right\| \leqq O\left(\lambda^{-\eta}\right) . \\
& \text { If } \varepsilon_{1} \geqq 1 / 2, \varepsilon_{2}=0, \lambda_{v}=0, \text { and } v \in A \\
& \left\|Q_{v}\right\| ! O\left(\log ^{2 N}[\text { largest upper cutoff in } \Delta]\right) \\
& \quad \leqq O\left(\log ^{2 N} n(\Delta)\right) .
\end{aligned}
$$

Let $s_{\mu}$ be the time of the $P$ vertex $\mu$ in $R_{\mu}$, then there exists $\eta>0$ such that

$$
\int d s_{\mu}\left\|R_{\mu}\right\|_{2} \leqq O\left(\lambda_{\mu}^{-\eta}\right)
$$

uniformly in the times of the other vertices not already integrated out. The $d s_{\mu}$ integration is between the times of the $P$ vertices introduced into the square containing $\mu$ immediately before and after $\mu$.

Whenever $v$ is a vertex with no open lines (excluding vertices in $R$ terms), apply (5.2). If $v$ has an open line, apply (5.3). To $R$ terms apply (5.4).

The time integral is now estimated for restricted values of the $\sigma_{i}$. The range of integration time orders the $P$ vertices, but not the $C$ vertices. Overestimate by discarding the time ordering of the $P$ vertices. The integral now factors into subintegrals corresponding to connected subgraphs of the fermion graph specified by $g$. These are either closed loops or lines. The $R$ terms have already been estimated by (5.4). The remaining loops with two vertices have $d_{l}>0$, hence $\sigma_{l}=0$ for both lines, hence these subintegrals are estimated by $O(1)$. Subintegrals corresponding to loops with $n$ vertices are less than $O(1)^{n}$ provided each $\sigma_{l}<2 / 3$, (which is the condition that a loop with three vertices give a convergent time integral. Loops with more vertices are better behaved). A subintegral corresponding to a connected open line with $n$ vertices is less than $O(1)^{n}$ provided each $\sigma_{l}<1$. These assertions are easy to verify. The restrictions of the above paragraph and (5.2) and (5.3) are all satisfied by the following choices: If $l$ with $d_{l}=0$ connects two vertices, neither of which have open lines, let $\sigma_{l}=5 / 8, \varepsilon_{-}=\varepsilon_{+}=5 / 17$. If $l$ with $d_{l}=0$ connects two vertices and the one at the " + " and of the line has an open line, let $\sigma_{l}=13 / 16$, $\varepsilon_{-}=5 / 17, \varepsilon_{+}=1 / 2$. If $l$ is an open line with the vertex at the " - " end, let $\sigma_{l}=\varepsilon_{-}=0$. If $l$ is any line with $d_{l}>0$, let $\sigma_{l}=0, \varepsilon_{+}=\varepsilon_{-}=1$. Choose $\eta>0, \eta<5 / 16-5 / 17$. 
By (5.1)-(5.4) and the paragraph above

$$
\left\langle e^{-T H}\right\rangle \leqq \sum_{g}\left(\prod_{\Delta} f(\Delta)\right) \prod_{v}\left(1+\lambda_{v}\right)^{-\eta} \prod_{l}\left(1+d_{l}\right)^{-m}
$$

for some $\eta>0$ and all $m \geqq 0$. $f(\Delta)$ is defined by

$$
f(\Delta)=\left[O(1) n(\Delta) \log ^{2} n(\Delta)\right]^{N n(\Delta)}
$$

for $O(1)$ a suitably large constant. $v$ now runs over all vertices. Let $n(\cdot)$ denote a function $\Delta \mapsto n(\Delta)$ and let $\{g: n(\cdot)\}$ be the set of all $g$ with the same given $n(\cdot)$ Rewrite (5.7) as

$$
\left\langle e^{-T H}\right\rangle \leqq \sum_{n(\cdot)}\left(\prod_{\Delta} f(\Delta)\right) \sum_{\{g} \prod_{n}\left(1+\lambda_{v}\right)^{-n} \prod_{l}\left(1+d_{l}\right)^{-m} .
$$

Estimate the second sum by

Lemma. Let $F=F(g)$, then

$$
\sum_{\{g: n(\cdot)} F(g) \prod_{l}\left(1+d_{l}\right)^{-3} \leqq\left[\prod_{d} O(1)^{n(d)}(2 n(\Delta)) !\right] \sup _{\{g: n(\cdot)\}} F(g)
$$

where l runs over all lines specified by g.

For a detailed proof of this kind of lemma, see [7], lemma (2.6). Briefly: Let $\tilde{g}$ denote the equivalence class of all $g$ with (1) $n(\cdot)$ specified. (2) The $P$ vertices which are part of $R$ subgraphs specified. (3) The vertices with an open line specified. (4) The localisations of the ends of each line not in an $R$ subgraph specified. The number of $g$ in $\tilde{g}$ is less than $\prod_{\Delta}(2 n(\Delta)) ![2 n(\Delta)$ ! overcounts the number of ways of assigning lines ending in $\Delta$ to vertices in $\Delta$.$] The factor \prod_{l}\left(1+d_{l}\right)^{-3}$ controls the sum over (4) because a line of length $d_{l}$ can contract to at most $O(1) d_{l}$ squares. $O(1)^{n(A)}$ controls the sum over (2) and (3).

Define $d_{v}$ to be 1 if $v$ is a $P$ vertex; if $v$ is a $C$ vertex, let $d_{v}$ be the sum of the $d_{l}$ 's for the lines $l$ joining $v$ to the $P$ vertex generating $v$. A $C$ vetex $v$ is said to be generated by a $P$ vertex $v^{\prime}$ if it was formed by contracting $v^{\prime}$ or by contracting a $C$ vertex which itself was formed by contracting $v^{\prime}$. Define $\lambda_{v}^{\prime}=\lambda_{v}$ if $v$ is a $P$ vertex, $=\lambda_{v^{\prime}}$ if $v$ is a $C$ vertex. $v^{\prime}$ is the $P$ vertex generating $v$. In terms of these definitions (5.9) and (5.10) imply, after relabelling $m$ and $\eta$ and the $O(1)$ in $f(A)$

$$
\begin{aligned}
\left\langle e^{-T H}\right\rangle \leqq & \sum_{n(\cdot)} \prod_{\Delta} f(\Delta)(2 n(\Delta)) ! \\
& \cdot \sup _{\{g: n(\cdot)\}} \prod_{v} \lambda_{v}^{\prime-\eta} d_{v}^{-m} \\
\leqq & \prod_{\Delta} \sum_{n(\Delta)} f(\Delta) 2 n(\Delta) ! \\
& \cdot \sup _{\{g: n(\Delta)\}} \prod_{v \in \Delta} \lambda_{v}^{\prime-n} d_{v}^{-m}
\end{aligned}
$$

$\{g: n(\Delta)\}$ is the set of all $g$ with the same $n(\Delta)$ in a given $\Delta$. It is now proved that if $\alpha$ is sufficiently large and if $m$ is sufficiently large depending on $\alpha$ then

$$
\sup _{\{g: n(\Delta)\}} \prod_{A} \lambda_{v}^{\prime-\eta} d_{v}^{-m} \leqq O(1)^{n(\Delta)} n(\Delta)^{-(N+3) n(\Delta)}
$$


(5.13) and (5.12) combine to give

$$
\left\langle e^{-r H}\right\rangle \leqq \prod_{\Delta} O(1)=O(1)^{|\mathrm{supp} g|}
$$

This concludes the estimation of (1.1) for the special case of the Fock vacuum expectation and, as explained in the introduction and Appendix III, demonstrates boundedness below linearly in the volume for the Hamiltonian.

Proof of (5.13). Fix $\Delta$. Let $C_{i}$ be the set of all vertices in $\Delta$, excluding $C$ vertices in $R$ terms, with $d_{v}=i$. Let $\left|C_{i}\right|$ denote the number of vertices in $C_{i}$. The expansion has been constructed so that if $n P$ vertices are localised in the same square, they must all have different cutoffs chosen from the sequence $f^{x}, j=1,2, \ldots$. Consequently the product of factors $\lambda_{v}^{-\eta}$ for these vertices is less than $(n !)^{-\eta \alpha}$. The $P$ vertices generating vertices in $C_{i}$, by definition of $C_{i}$, are localised in less than $25 i^{2}$ squares. The product of factors $\lambda_{v}^{-\eta}$ for these $P$ vertices can easily be vertified to be overestimated by $\left[\left(\left|C_{i}\right| / 100 i^{2}\right) !\right]^{25 i^{2} \eta \alpha}$. Therefore for all $g$

$$
\begin{aligned}
\prod_{v \in A} \lambda_{v}^{\prime-\eta} d_{v}^{-m} & \leqq \prod_{i} i^{-m\left|C_{i}\right|} \prod_{v \in C_{i}} \lambda_{v}^{\prime-\eta} \\
& \leqq \prod_{i} i^{-m\left|C_{i}\right|}\left[\left(\left|C_{i}\right| / 100 i^{2}\right) !\right]^{-25 i^{2} \eta \alpha} .
\end{aligned}
$$

The $\left|C_{i}\right|$ are constrained by

$$
\sum_{i}\left|C_{i}\right|=n(\Delta)
$$

The log of the right hand side of (5.15) is less than

$$
J=-\sum_{i}\left[m\left|C_{i}\right| \log i+\eta \alpha / 4\left|C_{i}\right|\left(\log \left|C_{i}\right|\left(100 i^{2}\right)^{-1}-1\right)\right] .
$$

The supremum of $J$ with respect to $\left|C_{i}\right|$ constrained by (5.16) occurs when (5.16) holds and, for each $i$

$$
m \log i+\eta \alpha / 4 \log \left|C_{i}\right|\left(100 i^{2}\right)^{-1}=\lambda .
$$

$\lambda$ is a Lagrange multiplier. Therefore

$$
\sup J=-\sum_{i}\left|C_{i}\right|(\lambda-\eta \alpha / 4)=-n(\Delta)(\lambda-\eta \alpha / 4)
$$

and (5.18) and (5.16) imply

$$
\lambda=\eta \alpha / 4\left[\log n(\Delta)-\log \sum_{i} 100 i^{2-4 m / \eta \alpha}\right] .
$$

Choose $\alpha$ so large that $\eta \alpha / 4 \geqq N+3$. Choose $m$ so large that $2-4 m / \eta \alpha \leqq-2$. Then the sum in (5.20) converges and thence

$$
\lambda \geqq(N+3) \log n(\Delta)-O(1)
$$

Equations (5.21) and (5.19) imply (5.13). Proof of (5.13) concluded. 


\section{Estimates on the Exponent}

This section and the next one are concerned with the justification of (4.3) and (4.5), namely: to show there is a function of Nelson fields $c(\phi)$ so that

$$
\begin{aligned}
& H_{0 F}(\tau)+\sum_{\Delta} V\left(\Delta, s_{g}, \tau\right) \geqq-c(\phi) \\
& \left\langle e^{2 \int c(\phi) d \tau}\right\rangle^{1 / 2} \leqq \prod_{\Delta} O(1)^{n(\Delta)} .
\end{aligned}
$$

$c(\phi)$ will depend on $\tau$ and $s_{\mathscr{g}}$ but (6.2) is uniform in $s_{\mathscr{g}}$. For each $\Delta, V\left(\Delta, s_{\mathscr{g}}, \tau\right) \equiv$ $V(\Delta)$ is split into four parts. $(\Lambda(\Delta) \equiv \Lambda(\Delta, \tau))$

$$
V(A)=V_{p}(\Delta)+V_{s}(\Delta)-A(A)+\int h \chi_{\Delta}: \phi^{2 M}: d x
$$

where, letting $\varrho \equiv \varrho\left(\Delta, \tau, s_{g}, p_{1}, p_{2}\right)$ denote the momentum cutoff in the exponent in $\Delta$,

$$
\begin{aligned}
& V_{p}(\Delta)=\int d p_{1} d p_{2} \mathscr{K}_{p} \varrho\left[b^{*}\left(p_{1}\right) b^{*}\left(p_{2}\right)+b\left(-p_{1}\right) b^{\prime}\left(-p_{2}\right)\right]\left(: \phi_{\Delta}^{N}:\right)^{\sim}\left(p_{1}+p_{2}, \tau\right) \\
& V_{s}(\Delta)=\int d p_{1} d p_{2} \mathscr{K}_{s} \varrho\left[b^{*}\left(p_{1}\right) b\left(-p_{2}\right)+b^{*}\left(p_{1}\right) b^{\prime}\left(-p_{2}\right)\right]\left(: \phi_{\Delta}^{N}:\right)^{\vee}\left(p_{1}+p_{2}, \tau\right) .
\end{aligned}
$$

The dummy time dependence of the fermion fields is dropped. The remaining estimates in this section will all be pointwise in time. $A$ has been defined in (2.11). Define, for $\tau^{\prime}<1, \tilde{\omega}=\omega-l \omega^{\tau^{\prime}}$ where $l>0$ is chosen so that $\tilde{\omega}>0$ and let

$$
N_{\tau^{\prime} F}=\int \omega^{\tau^{\prime}}(p)\left[b^{*}(p) b(p)+b^{*}(p) b^{\prime}(p)\right] d p
$$

Estimates (6.1) and (6.2) are obtained by finding functions $c_{p}(\phi)$ and $c_{s}(\phi)$ satisfying:

$$
\begin{aligned}
& H_{0 F}-l N_{\tau^{\prime} F}+\sum_{\Delta}\left[V_{p}(\Delta)-A(\Delta)\right] \geqq-c_{p}(\phi) \\
& l N_{\tau^{\prime} F}+\sum_{\Delta} V_{s}(\Delta) \geqq-c_{s}(\phi) \\
& \left\langle e^{2 \int\left[c_{p}(\phi)+c_{s}(\phi)\right] d \tau-2 j h: \phi^{2 M}: d x d \tau}\right\rangle^{1 / 2} \leqq \prod_{A} O(1)^{n(\Delta)} .
\end{aligned}
$$

The remainder of this section is to choose $c_{p}$ and $c_{s}$ and verify (6.8) and (6.9). (6.10) is discussed in the next section.

$c_{p}(\phi)$ is produced by Glimm's dressing transformation. Let

$$
\begin{aligned}
\Gamma V_{p}= & \sum_{\Delta} \int d p_{1} d p_{2} \mathscr{K}_{p} \varrho\left(\tilde{\omega}_{1}+\tilde{\omega}_{2}\right)^{-1} \\
& \cdot\left[b^{*}\left(p_{1}\right) b^{*}\left(p_{2}\right)-b\left(-p_{1}\right) b^{\prime}\left(-p_{2}\right)\right]\left(: \phi_{\Delta}^{N}:\right)^{\varkappa}\left(p_{1}+p_{2}, \tau\right) .
\end{aligned}
$$

$\Gamma V_{p}$ has been designed to satisfy

$$
\left[H_{O F}-\left|N_{t^{\prime} F}, \Gamma V_{p}\right|=\sum V_{p}(\Delta)\right. \text {. }
$$

Define

$$
\tilde{b}(p)=b(p)+\left[b(p), \Gamma V_{p}\right] ; \quad \tilde{b}^{\prime}(p)=b(p)+\left[b^{\prime}(p), \Gamma V_{p}\right]
$$


Calculate the positive operator $\int \tilde{\omega}(p)\left[\tilde{b}^{*}(p) \tilde{b}(p)+\tilde{b}^{*}(p) \tilde{b}^{\prime}(p)\right] d p$. Thence:

$$
H_{O F}-l N_{\tau^{\prime} F}+\sum_{\Delta} V_{p}(\Delta)+\int d p \tilde{\omega}\left[b^{*}(p), \Gamma V_{p}\right]\left[b(p), \Gamma V_{p}\right]+\left(\text { same term in } b^{\prime}\right) \geqq 0 .
$$

The two second order terms in (6.14) are positive operators, since the integrands have the form $A^{*} A$. The fermion annihilation and creation operators in these terms are antinormal ordered. On normal ordering, one obtains contracted terms and negative operators by virtue of the anticommutation rules. The negative operators can be discarded to obtain

$$
H_{O F}-l N_{\tau, F}+\sum_{\Delta} V_{p}(\Delta)+\sum_{\Delta, d^{\prime}} \tilde{A}\left(A, \Delta^{\prime}\right) \geqq 0 .
$$

The sum represents the contracted terms.

$$
\tilde{A}\left(\Delta, \Delta^{\prime}\right)=\int d k\left(: \phi_{\Delta}^{N}:\right)^{r}(k, \tau)\left[\int_{p_{1}+p_{2}=k} \mathscr{K}_{p}^{2} \varrho(\Delta) \varrho\left(\Delta^{\prime}\right)\left(\tilde{\omega}_{1}+\tilde{\omega}_{2}\right)^{-1]}\right]\left(: \phi_{\Delta^{\prime}}^{N}:\right)^{\prime}(k, \tau) .
$$

Therefore (6.8) hods for the choice

$$
c_{p}(\phi)=\sum_{d, A^{\prime}} \tilde{A}\left(\Delta, \Delta^{\prime}\right)+\sum_{d} A(\Delta) .
$$

The scattering part $V_{s}$ :

Let $\omega_{\tau^{\prime}, \text { LOC }}$ be the operator

$$
\left(1+x^{2}\right)^{-1} \omega^{\tau^{\prime}}(D)\left(1+x^{2}\right)^{-1}
$$

where $D=(1 / i) d / d x$. Define $N_{r^{\prime}, \text { LOC }}$ to be the Friedrichs extension of the second quantisation, with respect to fermions only, of $\omega_{\tau^{\prime}, \text { Loc }}$; the extension being from the domain of wavefunctions in Schwarz space describing finite numbers of particles. Let $N_{\tau^{\prime}, \text { LOC, } i}$ denote the translation of $N_{\tau^{\prime}, \mathrm{LOC}}$, in the obvious sense, by $i$ units.

Lemma. For $\tau^{\prime}<1$

$$
\sum_{i=-L}^{i=L} N_{\tau^{\prime}, \mathrm{LOC}, i} \leqq O(1) N_{\tau^{\prime} F}
$$

uniformly in L. For a proof, see Lemma (2.11) in [13].

Define

$$
\tilde{V}\left(p_{1}, p_{2}, q\right)=\left(\mathscr{K}_{s} \varrho\right)\left(p_{1}, p_{2}\right)\left(: \phi_{\Delta}^{N}:\right)^{\varkappa}\left(p_{1}+p_{2}, \tau\right)
$$

where $q$ denotes a point in $Q$ space. Then,

$$
V_{s}(\Delta)=\int d p_{1} d p_{2} \tilde{V}\left[b_{1}^{*} b_{2}+b_{1}^{*} b_{2}^{\prime}\right]
$$

letting $b_{1}^{*}=b^{*}\left(p_{1}\right), b_{2}=b\left(-p_{2}\right)$ etc. Observe that $\mathscr{K}_{s} \varrho \in S\left(\mathbb{R}^{2}\right)$ Schwarz space.

To obtain $c_{s}(\phi)$ begin by estimating, for $\Delta$ the square centred on the origin,

$$
\left\|N_{\tau^{\prime}, \mathbf{L O C}}^{-1 / 2} V_{s}(\Delta)\right\|_{F} \text {. }
$$

The subscript $F$ means that the norm is with respect to the fermion Fock space only, i.e., the total Fock space if fibered over $Q$ space. By a local $N_{\mathrm{r}}$ estimate. 
(See [13]), (6.24) is less than

$$
2\left\|\omega_{1}^{-\tau^{\prime} / 2}\left(1+D_{1}^{2}\right) \tilde{V}\right\|_{2} .
$$

The $L_{2}$ norm is with respect to the fermion variables in the kernel. The boson field is regarded as a numerical quantity. The subscripts 1 on $\omega$ and $D$ are to indicate that these operate on $p_{1}$ in $\tilde{V} .1+D^{2}$ is the fourier transform of $1+x^{2}$. Define

$$
W\left(p_{1}, p_{2}, q\right)=\left(1+D_{1}^{2}\right) \tilde{V}\left(p_{1}, p_{2}, q\right) .
$$

The estimate (6.25) for (6.24) implies

$$
\left\|N_{\tau^{\prime}, \text { LOC }}^{-1 / 2} V_{s}^{2}(4) N_{\tau^{\prime}, \text { LOC }}^{-1 / 2}\right\|_{F} \leqq 4\left\|\omega_{1}^{-\tau^{\prime} / 2} W\right\|_{2}^{2}
$$

which is the same as

$$
V_{s}^{2}(\Delta) \leqq 4 N_{\tau^{\prime}, \operatorname{LOC}}\left\|\omega_{1}^{-\tau^{\prime} / 2} W\right\|_{2}^{2}
$$

which implies

$$
\begin{aligned}
\pm V_{s}(\Delta) & \leqq 2 N_{\tau^{\prime}, \text { LOC }}^{1 / 2}\left\|\omega_{1}^{-\tau^{\prime} / 2} W\right\|_{2} \\
& \leqq \eta^{2} N_{\tau^{\prime}, \text { LOC }}+\left(1 / \eta^{2}\right)\left\|\omega_{1}^{-\tau^{\prime} / 2} W\right\|_{2}^{2}
\end{aligned}
$$

for any $\eta>0$. Estimate (6.29) is valid because operator inequalities are preserved on taking square roots. There are no technical difficulties with this because $V_{s}^{2}$ commutes with $N_{F}$, the fermion number operator, so $V_{s}^{2}$ can be proved to be self adjoint by restricting attention to eigenspaces of $N_{F}$.

Since the fermion momenta are restricted to lie below $\kappa_{n(\Delta)}$ by $\varrho$

$$
\begin{aligned}
\left\|\omega_{1}^{-\tau^{\prime} / 2} W\right\|_{2}^{2} & \leqq \omega\left(\kappa_{n(\Delta)}\right)^{\varepsilon}\left\|\omega_{1}^{-\tau^{\prime} / 2} \omega_{2}^{-\varepsilon / 2} W\right\|_{2}^{2} \\
& \leqq \delta^{-1}\left\|\omega_{1}^{-\tau^{\prime} / 2} \omega_{2}^{-\varepsilon / 2} W\right\|_{2}^{2 \delta}+(\delta-1) / \delta \omega\left(\kappa_{n(\Delta)}\right)^{\varepsilon \delta /(\delta-1)}
\end{aligned}
$$

for all $\varepsilon>0$ and $\delta>1$. The point of this is that while (6.25) diverges with $\kappa_{n(\Delta)}$, the kernel of the first term on the right of (6.31) does not diverge (with suitable choices of $\tau$ and $\varepsilon$ ). Choose $\delta>1$ so that $N \delta<M$. [These are the $N$ and $M$ in the expression for the interaction (2.2).] This is possible by the hypothesis $M>N$. Next choose $\varepsilon>0$ small so that $\varepsilon \delta /(\delta-1)<1 / \alpha$. Recall $\alpha$ was determined in Section 5 . As a consequence of this choice of $\varepsilon$

$$
\omega\left(\kappa_{n(\Delta)}\right)^{\varepsilon \delta /(\delta-1)} \leqq O(n(\Delta)) \text {. }
$$

Recall $\kappa_{n(\Delta)}=n(\Delta)^{\alpha}$. Next choose $\tau^{\prime}<1$ so that $\tau^{\prime}+\varepsilon>1$. This means that the kernel of the first term on the right of (6.31) is convergent independently of the cutoff. Clearly there are estimates analogous to (6.30) and (6.31) for each $\Delta$ and the choices of $\varepsilon, \delta, \tau^{\prime}$ are independant of $\Delta$. Therefore choose $\eta$ in (6.30) so small that on combining (6.30) and (6.31), summing over $\Delta$ and applying (6.21) one obtains

$$
\pm \sum_{\Delta} V_{s}(\Delta) \leqq l N_{t^{\prime} F}+O(1) \sum_{\Delta}\left(\left\|\omega_{1}^{-\tau^{\prime} / 2} \omega_{2}^{-\varepsilon / 2} W(\Delta)\right\|_{2}^{2 \delta}+n(\Delta)\right)
$$

Comparing (6.33) with (6.9) shows that $c_{s}$ may be chosen to be the second term on the right of (6.33). 


\section{The Estimate (6.10)}

In this section the proof of (6.1) and (6.2) is completed by proving (6.10).

First $c_{p}$ is majorised by a sum of terms each of which depend on boson fields localised in single squares. Define

$$
D\left(k, \Delta, \Delta^{\prime}\right)=\int_{p_{1}+p_{2}=k}\left|\mathscr{K}_{p}\left(p_{1}, p_{2}\right)\right|^{2} \varrho_{\Delta} \varrho_{\Delta^{\prime}}\left(\tilde{\omega}_{1}+\tilde{\omega}_{2}\right)^{-1} .
$$

Choose a function $\chi \in C^{\infty}(\mathbb{R})$ such that $\chi(t)=0$ for $t \leqq 1 / 2,=1$ for $t \geqq 1$. Define $\chi_{\Delta, \Delta^{\prime}}(x)=\chi\left(|x| / \operatorname{dist}\left(\Delta, A^{\prime}\right)\right),=1$ if $\operatorname{dist}\left(\Delta, A^{\prime}\right)=0$. Define $D^{\prime}\left(k, A^{\prime} A^{\prime}\right)=(2 \pi)^{-1 / 2} D *$ $\chi_{\Delta, \Delta^{\prime}}\left(k, \Delta, \Delta^{\prime}\right)$. Then

$$
\begin{aligned}
\tilde{A}\left(\Delta, \Delta^{\prime}\right) & =\int\left(: \phi_{\Delta}^{N}:\right)^{\wedge} D^{\prime}\left(: \phi_{\Delta^{\prime}}^{N}:\right)^{2} d k \\
& \leqq\left(\int\left|\left(: \phi_{\Delta}^{N}:\right)^{\prime}\right|^{2}\left|D^{\prime}\right| d k\right)^{1 / 2}\left(\int\left|\left(: \phi_{\Delta^{\prime}}^{N}\right)^{\prime}\right|^{2}\left|D^{\prime}\right| d k\right)^{1 / 2} \\
& \leqq \frac{1}{2} \int\left|\left(: \phi_{\Delta}^{N}:\right)^{\prime}\right|^{2}\left|D^{\prime}\right| d k+\frac{1}{2} \int\left|\left(: \phi_{\Delta^{\prime}}^{N}:\right)^{\prime}\right|^{2}\left|D^{\prime}\right| d k .
\end{aligned}
$$

Therefore by (6.17)

$$
c_{p}(\phi) \leqq \sum_{\Delta}\left\{\int\left|\left(: \phi_{\Delta}^{N}:\right)^{\prime}\right|^{2}\left(\sum_{\Delta^{\prime}}\left|D^{\prime}\left(\Delta, \Delta^{\prime}, k\right)\right|\right) d k+\Lambda(\Delta)\right\} .
$$

Split the sum over $A^{\prime}$ by setting

$$
\sum_{A^{\prime}}\left|D^{\prime}\right|=\sum_{\Delta^{\prime} \in \mathcal{N}(\Delta)}\left|D^{\prime}\right|+\sum_{\Delta^{\prime} \notin \mathscr{H}(\Delta)}\left|D^{\prime}\right| .
$$

Recall that $\mathcal{N}(\Delta)$ is the set of $\Delta^{\prime}$ within distance zero of $\Delta$. If $\operatorname{dist}\left(\Delta, \Delta^{\prime}\right)>0$

$$
\left|D^{\prime}(k)\right| \leqq O(1) \mu^{-1}(k) \operatorname{dist}\left(\Delta, \Delta^{\prime}\right)^{-3} .
$$

This is an easy consequence of the rapid decay of the propagator and its derivatives (provided any cutoffs included in the propagator are smooth) away from the origin. Equation (7.5) is uniform in cutoffs. Therefore

$$
\sum_{\Delta^{\prime} \notin \mathcal{N}(\Delta)}|D|^{\prime} \leqq O(1) \mu^{-1}(k)
$$

uniformly in $\Delta$. From (7.6) and (7.3)

$$
c_{p}(\phi) \leqq \sum_{\Delta}\left\{\int\left|\left(: \phi_{\Delta}^{N}:\right)^{\wedge}\right|^{2}\left(\sum_{\Delta^{\prime} \in \mathcal{A}(\Delta)}|D|+O(1) \mu^{-1}\right) d k+\Lambda(\Delta)\right\} .
$$

The $D$ 's and $\Lambda(\Lambda)$ in (7.7) diverge as the cutoffs in $\varrho_{\Delta}, \varrho_{\Delta^{\prime}}$ tend to $\infty$. The same type of estimate as that used in the treatment of $V_{s}[\mathrm{see}(6.31)]$ is now used to majorise (7.7) by, $(\delta>1, \varepsilon>0)$

$$
\begin{aligned}
& O(1) \sum_{\Delta}\left\{\int \mid\left(: \phi_{\Delta}^{N}:\right)^{2}(k, \tau) \int_{p_{1}+p_{2}=k}\left(\tilde{\omega}_{1}+\tilde{\omega}_{2}\right)^{-1} \omega_{1}^{-\varepsilon} d k\right\}^{\delta} \\
& \quad+\sum_{\Delta}\left[O(n(\Delta))+\left|\Lambda(\Delta) / k_{n(\Delta)}^{\varepsilon}\right|^{\delta}\right] .
\end{aligned}
$$

It is now shown that for $\lambda>0$ and $1 \leqq p<\infty$

$$
\left\|\exp \left[\int d \tau c_{s}(\phi)-\lambda \int h: \phi^{2 M}: d x d \tau\right]\right\|_{p} \leqq \prod_{\Delta} O(1)^{n(\Delta)} .
$$


The same type of proof applies to the terms in (7.8). The resulting estimate can be combined with (7.9) by the Holder inequality to complete the proof of (6.10).

The first step is to apply (4.13). Thus (7.9) is implied by

$$
\left\|\exp \left[\int d \tau \| \omega_{1}^{-\tau^{\prime} / 2} \omega_{2}^{-\varepsilon / 2} W(\Delta)\right]\right\|_{2}^{2 \delta}-\lambda \int h \chi_{\Delta}: \phi^{2 M}: d x d \tau \|_{p} \leqq O(1)
$$

uniformly in $\Delta . p$ and $\lambda$ have been relabelled. The method of proof is that in Nelson's original proof of boundedness below for $P(\phi)_{2}$. (See [10].) Let

$$
\mathscr{U}=\left\|\omega_{1}^{-\tau^{\prime} / 2} \omega_{2}^{-\varepsilon / 2} W(\Delta)\right\|_{2}^{2} ; \quad \mathscr{W}=\int d x d \tau h \chi_{\Delta}: \phi^{2 M}: d x
$$

These are both monomials in Nelson fields. Define $\mathscr{U}_{L}, \mathscr{W}_{L}$ by substituting $\phi \rightarrow \phi_{L}$ in (7.11). $\phi_{L}=(2 \pi)^{-1 / 2} \xi(|(\cdot)| / L) * \phi$, a field with momentum cut off above $L$. For all $L \geqq 0$

$$
\int d \tau\left(\mathscr{U}^{\delta}-\lambda \mathscr{W}\right) \leqq \int\left(\mu \mathscr{U}_{L}^{\delta}-\lambda \mathscr{W}_{L}\right) d \tau+\int d \tau\left[\mu\left|\mathscr{U}-\mathscr{U}_{L}\right|^{\delta}-\lambda\left(\mathscr{W}-\mathscr{W}_{L}\right)\right]
$$

recall $\delta>1$. $\mu$ is a constant depending only on $\delta$. Nelson's proof can be applied using (7.12) provided, for some $\gamma$ and $\eta>0$,

$$
\begin{aligned}
& \mu \mathscr{U}_{L}^{\delta}-\lambda \mathscr{W _ { L }} \leqq O(1) \log ^{\gamma} L \\
& \left\|\int d \tau\left[\mu\left|\mathscr{U}-\mathscr{U}_{L}\right|^{\delta}-\lambda\left(\mathscr{W}-\mathscr{W}_{L}\right)\right]\right\|_{q} \leqq O(1) q^{\gamma} L^{-\eta}
\end{aligned}
$$

uniformly in $\Delta, \varrho(\Delta)$ and $q>1$.

Proof of (7.13).

$$
\mathscr{U}_{L}=\int d p_{1} d p_{2}\left|\left(1+D_{1}^{2}\right) \mathscr{K}_{s} \varrho\left(g \chi_{\Delta}: \phi_{L}^{N}\right)^{\wedge}\left(p_{1}+p_{2}, \tau\right)\right|^{2} \omega_{1}^{-\tau^{\prime}} \omega_{2}^{-\varepsilon}
$$

Perform the derivatives with respect to $p_{1}$ using Leibniz's rule. A typical term in the resulting sum is, using primes to indicate differentiation.

$$
\int d p_{1} d p_{2}\left(\mathscr{K}_{s} \varrho\right)^{\prime}\left(g \chi_{\Delta}: \phi_{L}^{N}:\right)^{\prime \prime}\left(\mathscr{K}_{s} \varrho\right)^{\prime \prime}\left(g \chi_{\Delta}: \phi_{L}^{N}:\right)^{2} \omega_{1}^{-\tau^{\prime}} \omega_{2}^{-\varepsilon}
$$

Define

$$
D(k)=\int_{p_{1}+p_{2}=k}\left(\mathscr{K}_{s} \varrho\right)^{\prime}\left(\mathscr{K}_{s} \varrho\right)^{\prime \prime} \omega_{1}^{-\tau^{\prime}} \omega_{2}^{-\varepsilon}
$$

so that (7.16) can be rewritten as

$$
(2 \pi)^{-1 / 2} \int x\left(g \chi_{\Delta}: \phi_{L}^{N}:\right)(x) \hat{D}(x-y)\left(g \chi_{A}: \phi_{L}^{N}:\right)(y) d x d y .
$$

Now it is easy to show that $|D(k)| \leqq$ const independantly of $\varrho$ because $\tau^{\prime}+\varepsilon>1$ and $\mathscr{K}_{s} \varrho$ and derivatives are bounded uniformly in $\varrho$. Thus the operator with kernel $x \chi_{A}(x) \hat{D}(x-y) \chi_{A}(y)$ is bounded and (7.18) is bounded in absolute value by

$$
O(1) \int d x\left(g \chi_{\Delta}: \phi_{L}^{N}:\right)^{2}(x)
$$

and in fact

$$
\mathscr{U}_{L} \leqq O(1) \int d x\left(g \chi_{\Delta}: \phi_{L}^{N}:\right)^{2}
$$

therefore

$$
\mathscr{U}_{L}^{\delta} \leqq O(1) \int d x\left(g \chi_{A}\right)^{2 \delta}\left(: \phi_{L}^{N}:\right)^{2 \delta} \leqq O(1) \int d x h \chi_{A}\left(: \phi_{L}^{N}:\right)^{2 \delta}
$$


using the Holder inequality followed by the hypotheses (2.3). So (7.13) is implied by

$$
O(1)\left(: \phi_{L}^{N}:\right)^{2 \delta}-\lambda: \phi_{L}^{2 M}: \leqq O(1) \log ^{\nu} L \text {. }
$$

This is implied by Wick's theorem. Recall $2 N \delta<2 M \delta$. End of proof of (7.13).

Proof of (7.14).

$$
\begin{aligned}
& \left\|\int d \tau\left[\mu\left|\mathscr{U}-\mathscr{U}_{L}\right|^{\delta}-\lambda\left(\mathscr{W}-\mathscr{W}_{L}\right)\right]\right\|_{q} \\
& \leqq \mu \int_{\Delta} d \tau\left\|\mathscr{U}-\mathscr{U}_{L}\right\|_{\delta q}^{\delta}+\lambda \int_{\Delta} d \tau\left\|\mathscr{W}-\mathscr{W}_{L}\right\|_{q} .
\end{aligned}
$$

The $\Delta$ on the time integral means that the integration is to extend over the corresponding time interval.

$$
\left\|\mathscr{U}-\mathscr{U}_{L}\right\|_{\delta q}^{\delta} \leqq(\delta q)^{N \delta}\left\|\mathscr{U}-\mathscr{U}_{L}\right\|_{2}^{\delta} \leqq(\delta q)^{N \delta} O(1) L^{-\eta} .
$$

The first inequality in (7.24) is Nelson's Best Estimate [11]. (An $N_{\tau}$ estimate is also sufficient.) The second inequality is valid for $\eta>0$ satisfying $\tau^{\prime}+\varepsilon-\eta / \delta>1$ and is not difficult to prove. A similar process applied to $\left\|\mathscr{W}-\mathscr{W}_{L}\right\|$ completes the proof of (7.14).

\section{Appendix I}

Estimate (5.4): $R_{\mu}$ is written as a sum of terms, each corresponding to one of the cases below. The $L_{2}$ norm of each is bounded separately. The notation used in "renormalisation" Section 3, is resurrected.

Case 1. $\Delta_{P}=\Delta_{C}=\Delta . s_{C}<s_{n-1}$

$$
\begin{array}{r}
\int d s_{C} \int d k\left(: \phi_{\Delta}^{N}:\right)^{\top}\left(s_{P}\right) r\left(: \phi_{\Delta}^{N}:\right)^{\top}\left(s_{C}\right) \leqq \int d s_{C}\left(\int r\left|\left(: \phi_{\Delta}^{N}:\right)^{\top}\right|^{2}\left(s_{P}\right) d k\right)^{1 / 2} \\
\cdot\left(\int r\left|\left(: \phi_{\Delta}^{N}:\right)^{\top}\right|^{2}\left(s_{C}\right) d k\right)^{1 / 2} .
\end{array}
$$

The range of the integration over $s_{C}$ is $s_{C}<s_{n-1}$. For $N=1$,

$$
\begin{aligned}
& \left\|\int r\left|\left(: \phi_{\Delta}^{N}:\right)^{\wedge}\right|^{2} d k\right\|_{2} \leqq O(1)\left[\int d k_{1} d k_{2} \mu_{1}^{-1} \mu_{2}^{-1}\right. \\
& \left.\left.\quad \cdot \int d k\left(g \chi_{\Delta}\right)^{(}\left(k_{1}-k\right) r(k)\left(g \chi_{A}\right)^{2}\left(k_{2}-k\right)\right|^{2}\right]^{1 / 2} \\
& \quad+O(1) \int d k\left|\left(g \chi_{\Delta}\right)^{2}\right|^{2}(k) \mu^{-1}(k) r(k) \\
& \leqq O(1) \int d k\left|\left(g \chi_{A}\right)^{2}\right|^{2} \mu^{-1}(k) r(k) .
\end{aligned}
$$

The norm on the left hand side of (A.2) is on $L^{2}(Q)$. The estimate (A.3) is by the Cauchy Schwarz inequality. A similar estimate holds for $N>1$. The right hand side of (A.3) may be estimated by combining the hypotheses (2.3) with

$$
r(k) \leqq O(1) \kappa_{n}^{-\eta}\left|s_{P}-s_{C}\right|^{-1-\varepsilon}
$$

valid for $\eta, \varepsilon$ such that $\varepsilon>\eta>0$. Estimate (A.4) is an easy consequence of (3.11). The factor $\left|s_{p}-s_{c}\right|^{-1-\varepsilon}$ is integrable for suitable choices of $\eta>0, \varepsilon>0$ because $s_{C}<s_{n-1}<s_{P}$. Collecting (A.1) to (A.4) proves that the integral with respect to $s_{P}$ of the $L^{2}(Q)$ norm of the left hand side of (A.1) is bounded by $O\left(\kappa_{n}^{-\eta}\right)$ for some $\eta>0$, uniformly in $s_{n-1}$. Case 1 is complete.

Case 2. $A_{P}=A_{C}=A . s_{n-1}<s_{C}<s_{P}$. 
While this part of $R_{\mu}$ does not diverge, (the $C$ vertex has an upper cutoff) it is still necessary to cancel it against the appropriate part of the counterterm to obtain the small $\lambda_{\mu}^{-n}$ convergence factor. The term under consideration is

$$
\int d s_{C} \int d k\left(: \phi_{\Delta}^{N}:\right)^{\Upsilon}\left(s_{p}\right) r\left(: \phi_{A}^{N}:\right)^{\Upsilon}\left(s_{C}\right) \text {. }
$$

In the above region of integration for $s_{C}$,

$$
\begin{gathered}
r\left(k, s_{P}, s_{C}\right) \equiv q\left(k, s_{P}-s_{C}\right)=\int_{p_{1}+p_{2}=k}\left|\mathscr{K}_{p}\right|^{2}\left[1-\xi\left(p_{1} / \kappa_{n}\right) \xi\left(p_{2} / \kappa_{n}\right)\right] \\
\cdot \xi\left(p_{1} / \kappa_{n}\right) \xi\left(p_{2} / \kappa_{n}\right) \exp \left(-\left[\omega_{1}+\omega_{2}\right]\left(s_{p}-s_{C}\right) .\right.
\end{gathered}
$$

Normal order the bosons in (A.5). A term in the resulting sum has the form

$$
\int d s_{C} \int d k:\left(\phi_{\Delta}^{N-j}\right)\left(s_{P}\right) q_{j}\left(\phi_{\Delta}^{N-j}\right)^{\sim}\left(s_{C}\right):
$$

$j=0,1, \ldots . N$, and

$$
q_{j}\left(k, s_{P}-s_{C}\right)=\text { const } \times \int_{l_{0}+\ldots+l_{j}=k} \prod_{i=1, \ldots, j} \mu\left(l_{i}\right)^{-1} \exp \left[-\mu\left(l_{i}\right)\left|s_{P}-s_{C}\right|\right] q\left(l_{0}\right) .
$$

The conterterm for (A.7) is

$$
\left[\int_{0}^{\infty} d t q_{j}(0, t)\right] \int d x\left(: \phi_{\Delta}^{2(N-j)}\right)\left(s_{P}\right) \text {. }
$$

The result of combining (A.7) and (A.9) can be written as the sum of

$$
\int d k:\left|\left(\phi_{\Delta}^{N-j}\right)\right|^{2}\left(s_{p}\right):\left(\int d s_{C} q_{j}\left(k, s_{p}-s_{\mathcal{C}}\right)-\int_{0}^{\infty} d t q_{j}(0, t)\right)
$$

and

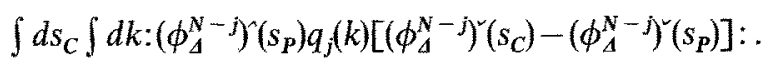

The $L^{2}(Q)$ norm of (A.10) is easily estimated by using

$$
\begin{aligned}
& \left|\int d s_{C} q_{j}\left(k, s_{P}-s_{C}\right)-\int_{0}^{\infty} d t q_{j}(0, t)\right| \\
& \leqq\left|\int_{0}^{\infty}\left(q_{j}(k, t)-q_{j}(0, t)\right) d t\right|+\int_{-\infty}^{s_{n}-1} q_{j}\left(k, s_{P}-s_{C}\right) d s_{C} \\
& \leqq O(1)(1+|k|)^{\varepsilon} \kappa_{n}^{-\eta}+O(1)\left|s_{P}-s_{n-1}\right|^{\varepsilon} \kappa_{n}^{-\eta}
\end{aligned}
$$

where $\varepsilon>\eta>0$ together with (2.3). The last line of (A.12) is tedious but elementary to derive. To estimate the $L^{2}(Q)$ norm of (A.11) write

$$
\left(\phi_{\Delta}^{N-j}\right)^{\wedge}\left(k_{1}, s\right)=(2 \pi)^{-1 / 2} \int d k_{0}\left(\phi_{\Delta}^{N-j}\right)^{\wedge}\left(k_{1}, k_{0}\right) e^{i k_{0} s}
$$

so that (A.11) becomes

$$
\begin{aligned}
& O(1) \int d k_{0} d k_{1} d l_{0}:\left(\phi_{A}^{N-j}\right)^{\wedge}\left(k_{1}, l_{0}\right)\left(\phi_{A}^{N-j}\right)^{\curlyvee}\left(k_{1}, k_{0}\right) \\
& \cdot e^{i\left(l_{0}-k_{0}\right) s_{P}} \int d s_{C} q_{j}\left(k_{1}, s_{P}-s_{C}\right)\left(e^{-i k_{0}\left(s_{C}-s_{P}\right)}-1\right) .
\end{aligned}
$$


Now use

$$
\begin{aligned}
& \left|e^{i\left(l_{0}-k_{0}\right) s_{P}} \int d s_{C} q_{j}\left(k_{1}, s_{P}-s_{C}\right)\left[e^{-i k_{0}\left(s_{C}-s_{P}\right)}-1\right]\right| \\
& \leqq O(1) \kappa_{n}^{-\eta}\left[\left|s_{P}-s_{n-1}\right|^{-\varepsilon}+\left(1+\left(k_{1}^{2}+k_{0}^{2}\right)\right)^{-\varepsilon / 2}\right]
\end{aligned}
$$

where $\varepsilon>\eta>0$, to estimate the $L^{2}(Q)$ norm of (A.14). End of Case 2.

Case 3. $\Delta_{P}=\Delta_{C}=\Delta . s_{P}<s_{C}$.

Repeat the argument in Case 2 with

$$
\begin{aligned}
q\left(k, s_{P}-s_{C}\right)= & \int_{p_{1}+p_{2}=k}\left|\mathscr{K}_{p}\right|^{2}\left(1-\xi\left(p_{1} / \kappa_{n}\right) \xi\left(p_{2} / \kappa_{n}\right)\right) \\
& \cdot \exp \left(-\left[\omega_{1}+\omega_{2}\right]\left|s_{P}-s_{C}\right|\right)
\end{aligned}
$$

which also satisfies (A.12) and (A.15). (The $C$ vertex now has no cutoff.) End of Case 3.

Case 4. $\Delta_{P} \neq \Delta_{C}$.

By normal ordering, the problem is reduced to estimating the $L^{2}(Q)$ norm of $\int d s_{C} \int d k:\left(\phi_{A_{p}}^{N-j}\right)^{\sim}\left(s_{p}\right) r_{j}\left(k, s_{p}, s_{C}\right)\left(\phi_{A_{C}}^{N-j}\right)^{2}\left(s_{C}\right):$

where

$$
\begin{aligned}
r_{j}\left(k, s_{P}, s_{C}\right)= & \text { const } \times \int_{l_{0}+\ldots+l_{j}=k} \prod_{i=1, \ldots, j} \mu\left(l_{i}\right)^{-1} \\
& \cdot \exp \left(-\mu\left(l_{i}\right) \mid s_{P}-s_{C}\right) r\left(l_{0}, s_{P}, s_{C}\right) .
\end{aligned}
$$

Observe that in (A.17), $r_{j}$, may be replaced by

$$
r_{j}^{\prime} \equiv r_{j}\left(k, s_{P}, s_{C}\right)-r_{j}\left(0, s_{p}, s_{C}\right)
$$

because the $P$ and $C$ vertices are localised in disjoint squares, so the second term in (A.19) makes no contribution. $r_{j}^{\prime}$ is not divergent. The norm of (A.17) can now be estimated using (A.13). End of Case 4.

\section{Appendix II}

The scattering part of $V_{s}$ can be estimated in another way using a "fractional local $N_{\tau}$ estimate". A formal derivation of this estimate is given below.

Suppose

$$
\begin{aligned}
& V=\int v\left(p_{1}, p_{2}\right) b^{*}\left(p_{1}\right) b\left(-p_{2}\right) d p_{1} d p_{2} \\
& w\left(p_{1}, p_{2}\right)=\left(1+D_{1}^{2}\right)\left(1+D_{2}^{2}\right) v\left(p_{1}, p_{2}\right) .
\end{aligned}
$$

Then for $\alpha>0$

$$
\left\|\left(1+N_{\tau, \mathrm{LOC}}\right)^{-1 / 2} V\left(1+N_{\tau, \mathrm{LOC}}\right)^{-\alpha / 2}\right\| \leqq O(1)\left[\int d p_{1} d p_{2} \omega_{1}^{-\tau} \omega_{2}^{-\varepsilon \tau}\left|w\left(p_{1}, p_{2}\right)\right|^{2}\right]^{1 / 2}
$$

with $\varepsilon<\alpha$, and $\varepsilon=\left(\frac{1}{2}\right)^{m}$ for some integer $m>0$.

Proof. Write

$$
\left(1+N_{\tau, \mathrm{LOC}}\right)^{-\alpha / 2}=O(1) \int_{0}^{\infty} d t t^{\alpha / 2-1} e^{-t\left(1+N_{\tau, \mathrm{LOC}}\right)} .
$$


Let $a=\omega_{\tau, \text { Loc }} \cdot a_{1}$ acts on $p_{1}, a_{2}$ on $p_{2}$. By (A.23) and the pull through formula, the left hand side of (A.22) is estimated by

$$
\begin{aligned}
& O(1) \int_{0}^{\infty} d t t^{\alpha / 2-1} \|\left(1+N_{\tau, \mathrm{LOC}}\right)^{-1 / 2} \int d p_{1} d p_{2}\left(e^{-t a_{2}} v\right)\left(p_{1}, p_{2}\right) \\
& \cdot b^{*}\left(p_{1}\right) e^{-t\left(1+N_{\tau, \mathrm{LOC}}\right)} b\left(-p_{2}\right) \| .
\end{aligned}
$$

By a local $N_{\tau}$ estimate (see [13]), (A.24) is less than

$$
O(1) \int_{0}^{\infty} d t t^{\alpha / 2-1} e^{-t}\left(\int d p_{1} d p_{2}\left|\left(\omega_{1}^{-\tau / 2}\left(1+D_{1}^{2}\right) e^{-t a_{2}} v\right)\right|^{2}\right)^{1 / 2} .
$$

The presence of the factor $e^{-t\left(1+N_{s, L O C}\right)}$ between the annihilation and creation operators in (A.24) does not disturb the proof of the local estimate given in [13], being a bounded operator. (The bound gives the $e^{-t}$.) By the estimate $\left\|a_{2}^{\varepsilon / 2} e^{-t a_{2}}\right\| \leqq$ $O(1) t^{-\varepsilon / 2},(\mathrm{~A} .25)$ is less than

$$
O(1) \int_{0}^{\infty} d t t^{\alpha / 2-1-\varepsilon / 2} e^{-t}\left(\int d p_{1} d p_{2}\left|\left(\omega_{1}^{-\tau / 2}\left(1+D_{1}^{2}\right) a_{2}^{-\varepsilon / 2} v\right)\right|^{2}\right)^{1 / 2}
$$

Provided $\alpha / 2-\varepsilon / 2>0$, Eq. (A.26) is less than

$$
O(1)\left(\int d p_{1} d p_{2}\left|\omega_{1}^{-\tau / 2}\left(1+D_{1}^{2}\right) a_{2}^{-\varepsilon / 2} v\right|^{2}\right)^{1 / 2} .
$$

Now obtain (A.22) by using the operator inequality

$$
a_{2}^{-\varepsilon} \leqq\left(1+D_{2}^{2}\right) \omega_{2}^{-\varepsilon \tau}\left(1+D_{2}^{2}\right)
$$

provided $\varepsilon=\left(\frac{1}{2}\right)^{m}$ for some $m \geqq 0$. To prove (A.28) observe that

$$
\left(\left(1+D^{2}\right) \omega^{-\tau}\left(1+D^{2}\right)\right)^{1 / 2} \leqq\left(1+D^{2}\right) \omega^{-\tau / 2}\left(1+D^{2}\right)
$$

is implied by (it can be proven that $\omega_{\tau, \operatorname{LOC}}$ is essentially self adjoint on $S(\mathbb{R})$ )

$$
\left(1+D^{2}\right) \omega^{-\tau}\left(1+D^{2}\right) \leqq(1+D)^{2} \omega^{-\tau / 2}\left(1+D^{2}\right)^{2} \omega^{-\tau / 2}\left(1+D^{2}\right)
$$

(operator inequalities are preserved by square roots) which is implied by

$$
\left(1+D^{2}\right)^{2} \geqq 1 \text {. }
$$

Iterating (A.30) and taking square roots gives (A.29).

\section{Appendix III: Uniformity in the Expectation}

It is sufficient to find a uniform bound for (1.1) when the state $s$ is a member of a dense subset, in particular when $S$ has the form of a sum of products of functions from $L^{\infty}(Q)$ in $\psi^{\#}(f) \mathrm{s},\|f\|_{2} \leqq 1 . \psi^{\#}$ denotes $\bar{\psi}$ or $\psi$. The lower bound for the right hand side of (1.1) with $S$ of this form is equal to the infimum over the cross product contributions so one is reduced to estimating

$$
-\lim _{T \rightarrow \infty} T^{-1} \log \left\langle s^{\prime} e^{-T H} s^{\prime \prime}\right\rangle
$$

where $s^{\prime}, s^{\prime \prime}$ are each products of an $L^{\infty}(Q)$ function with $\psi^{\#}(f)$ s. The $L^{\infty}$ functions can be majorised by their $L^{\infty}$ norms after defermiation. The constants so obtained 
make no contribution in the limit $T \rightarrow \infty$. The $\psi^{\#}(f) s$ which will be referred to as "external fields" cause a new class of fermion graphs because vertices can contract to them. The terms with these graphs still satisfy (4.12) so it is merely necessary to examine what change needs to be made in (5.10). Suppose there are $p$ external fields, then the extra graphs are claimed to be counted by prefacing the right hand side of $(5.10)$ by $\prod_{\Delta} 2^{n(\Delta)} p$ !. Clearly the $2^{n(\Delta)}$ can be absorbed into the $O(1)^{n(\Delta)}$ and the $p$ ! makes no contribution in the $T$ limit, so a uniform bound on (A.32) will hold. For each vertex, a factor 2 counts whether it contracts to an external field or not, hence the $\prod_{A} 2^{n(\Delta)}$. The $p$ ! counts the possible assignments of external fields to the vertices which contract externally.

\section{References}

1. Federbush, P.: Positivity for Some Generalised Yukawa Models in One Space Dimension, J. Math. Phys. 14, 1532-1542 (1973)

2. Federbush, P.: A New Approach to the Stability of Matter I. J. Math. Phys. 16, 347-351 (1975)

3. Federbush, P.: A New Approach to the Stability of Matter II. J. Math. Phys. 16, 706-709 (1975)

3.1. Federbush, P.: The Semi Euclidean Approach in Statistical Mechanics II. The Cluster Expansion. A special example. Preprint

4. Brydges, D., Federbush, P.: A Semi-Euclidean Approach to Boson Fermion Model Theories. J. Math. Phys. 15, 730-732 (1974)

5. Brydges, D.: Boundedness Below for Fermion Model Theories. Part I. J. Math. Phys. 16, 1649-1661 (1975)

6. Glimm, J.: Commun. Math. Phys. 5, 343-386 (1967); 6, 61-76 (1967)

7. Dimock, J., Glimm, J.: Measures on Schwartz Distribution Space and Applications to $P(\phi)_{2}$ Field Theories. N.Y.U. Preprint

8. Glimm, J., Jaffe, A.: Positivity and Self Adjointness of the $P(\phi)_{2}$ Hamiltonian. Commun. Math. Phys. 22, 253 (1971)

9. Glimm, J., Jaffe, A.: Statistical Mechanics and Quantum Field Theory. In: Les Houches Lecture Notes (C. DeWitt, R. Stora, eds.) New York: Gordon \& Breach 1970

10. Nelson, E.: A Quartic Interaction in Two Dimensions (R.Goodman, I.Segal, eds.) Cambridge: M.I.T. Press, 1966

11. Nelson, E.: The Free Markov Field, J. Funct. Anal. 12, 211-217 (1973)

12. Guerra, F., Rosen, L., Simon, B.: The $P(\phi)_{2}$ Euclidean Quantum Field Theory as Classical Statistical Mechanics. Ann. Math. 101, 111-259 (1975)

13. Schrader, R.: Yukawa Quantum Field Theory in Two Space Time Dimensions without Cutoffs. Ann. Phys. 70, 412-457 (1972)

14. McBryan, O.: Volume Dependence of Schwinger Functions in the Yukawa 2 Quantum Field Theory. Rockefeller University Preprint

15. Seiler,E.: Schwinger Functions for the Yukawa Model in Two Dimensions with Space-Time Cutoff. Commun. Math. Phys. 42, 163-182 (1975)

16. Seiler, E., Simon, B.: Bounds in the Yukawa ${ }_{2}$ Quantum Field Theory: Upper Bound on the Pressure, Hamiltonian Bound and Linear Lower Bound. Princeton University Preprint

Communicated by A. S. Wightman

(Received March 25, 1975; in revised form September 15, 1975) 